\title{
The AMBRE Project: Solar neighbourhood chemodynamical constraints on Galactic disc evolution
}

\author{
P. Santos-Peral, A. Recio-Blanco, G. Kordopatis, E. Fernández-Alvar, and P. de Laverny
}

\author{
Université Côte d'Azur, Observatoire de la Côte d'Azur, CNRS, Laboratoire Lagrange, Bd de l'Observatoire, CS 34229, \\ 06304 Nice cedex 4, France \\ e-mail: psantos@oca.eu
}

Received 16 December 2020 / Accepted 15 June 2021

\begin{abstract}
Context. The abundance of $\alpha$-elements relative to iron $([\alpha / \mathrm{Fe}])$ is an important fossil signature in Galactic archaeology for tracing the chemical evolution of disc stellar populations. High-precision chemical abundances, together with accurate stellar ages, distances, and dynamical data, are crucial to infer the Milky Way formation history.

Aims. The aim of this paper is to analyse the chemodynamical properties of the Galactic disc using precise magnesium abundance estimates for solar neighbourhood stars with accurate Gaia astrometric measurements.

Methods. We estimated ages and dynamical properties for 366 main sequence turn-off stars from the AMBRE Project using PARSEC isochrones together with astrometric and photometric values from Gaia DR2. We use precise global metallicities $[\mathrm{M} / \mathrm{H}]$ and $[\mathrm{Mg} / \mathrm{Fe}]$ abundances from a previous study in order to estimate gradients and temporal chemodynamic relations for these stars.

Results. We find a radial gradient of $-0.099 \pm 0.031 \mathrm{dex} \mathrm{kpc}^{-1}$ for $[\mathrm{M} / \mathrm{H}]$ and $+0.023 \pm 0.009 \mathrm{dex} \mathrm{kpc}^{-1}$ for the $[\mathrm{Mg} / \mathrm{Fe}] \mathrm{abundance}$. The steeper $[\mathrm{Mg} / \mathrm{Fe}]$ gradient than that found in the literature is a result of the improvement of the AMBRE $[\mathrm{Mg} / \mathrm{Fe}]$ estimates in the metal-rich regime. In addition, we find a significant spread of stellar age at any given $[\mathrm{Mg} / \mathrm{Fe}]$ value, and observe a clear correlated dispersion of the $[\mathrm{Mg} / \mathrm{Fe}]$ abundance with metallicity at a given age. While for $[\mathrm{M} / \mathrm{H}] \leq-0.2$, a clear age $-[\mathrm{Mg} / \mathrm{Fe}]$ trend is observed, more metal-rich stars display ages from 3 up to $12 \mathrm{Gyr}$, describing an almost flat trend in the $[\mathrm{Mg} / \mathrm{Fe}]-\mathrm{age}$ relation. Moreover, we report the presence of radially migrated and/or churned stars for a wide range of stellar ages, although we note the large uncertainties of the amplitude of the inferred change in orbital guiding radii. Finally, we observe the appearance of a second chemical sequence in the outer disc, $10-12$ Gyr ago, populating the metal-poor, low- $[\mathrm{Mg} / \mathrm{Fe}]$ tail. These stars are more metal-poor than the coexisting stellar population in the inner parts of the disc, and show lower $[\mathrm{Mg} / \mathrm{Fe}]$ abundances than prior disc stars of the same metallicity, leading to a chemical discontinuity. Our data favour the rapid formation of an early disc that settled in the inner regions, followed by the accretion of external metal-poor gas -probably related to a major accretion event such as the Gaia-Enceladus/Sausage one- that may have triggered the formation of the thin disc population and steepened the abundance gradient in the early disc.
\end{abstract}

Key words. Galaxy: disk - stars: abundances - Galaxy: evolution - Galaxy: kinematics and dynamics - methods: observational

\section{Introduction}

A thorough understanding of the formation and evolution of the Milky Way demands precise chemical abundances and stellar ages. The stellar upper atmospheres of non-evolved stars provide fossil evidence of the available metals of the interstellar medium (ISM) at the time of formation (Freeman \& Bland-Hawthorn 2002). The present-day observed chemical signatures in the Galactic stellar populations, together with ages, distances, and dynamical data, allow us to infer the different stages of the history of the Galaxy.

The formation of the Galactic disc is still not well understood and in particular the origin and existence of a thin-thick disc bimodality is a matter of debate. Following the initial thick disc identification from stellar density distributions (Yoshii 1982; Gilmore \& Reid 1983) and the first attempts to kinematically classify the stars as part of either the thin or the thick disc (Bensby et al. 2003, 2005; Reddy et al. 2006), the two Galactic disc populations in the solar neighbourhood are often distinguished based on their abundances of $\alpha$-elements (e.g., $\mathrm{Mg}, \mathrm{Si}, \mathrm{Ti}$ ) relative to iron (e.g., Adibekyan et al. 2012; Recio-Blanco et al. 2014;
Bensby et al. 2014; Kordopatis et al. 2015a, 2017; Wojno et al. 2016; Fuhrmann et al. 2017; Hayden et al. 2017; Minchev et al. 2018). The $[\alpha / \mathrm{Fe}]$ versus metallicity $[\mathrm{M} / \mathrm{H}]$ plane provides valuable clues as to the disc stellar population evolution. The thick disc is often reported to be $[\alpha / \mathrm{Fe}]$-enhanced relative to the thin disc, suggesting distinct chemical evolution histories. In particular, magnesium is often used as an $\alpha$-elements tracer (e.g., Fuhrmann 1998; Mikolaitis et al. 2014; Bergemann et al. 2014; Carrera et al. 2019) because there is a high number of measurable spectral lines in optical spectra, and it also clearly separates the chemical sequences of the discs. Additionally, observational studies have shown that the thick disc stellar population could have been formed on a short timescale before the epoch of the thin disc formation (Haywood et al. 2013; Kordopatis et al. 2015a; Fuhrmann et al. 2017; Silva Aguirre et al. 2018; Delgado Mena et al. 2019; Ciucă et al. 2021; Katz et al. 2021). This scenario is supported by two-infall chemical evolution models (Grisoni et al. 2017; Spitoni et al. 2019; Palla et al. 2020).

Moreover, the observed stellar radial abundance distribution and the age-abundance relations in the Galactic disc are interesting signatures for studying the chemical enrichment history. 
They contain information about the star formation efficiency at different Galactocentric distances and on different timescales (Magrini et al. 2009; Minchev et al. 2014; Anders et al. 2014), the radial migration of stars (Sellwood \& Binney 2002; Schönrich \& Binney 2009; Minchev et al. 2018), and the infall of gas (Oort 1970; Schönrich \& McMillan 2017; Grisoni et al. 2017). An inside-out formation scenario (Matteucci \& Francois 1989) combined with a higher star formation efficiency in the inner regions of the Galaxy seems to reproduce the present-day local abundance gradients (Grisoni et al. 2018), where metallicity decreases with Galactocentric radius. In addition, a tight correlation between $[\mathrm{Mg} / \mathrm{Fe}]$-enhancement and age has been found for the old thick disc population (e.g., Haywood et al. 2013; Bensby et al. 2014; Hayden et al. 2017; Delgado Mena et al. 2019; Nissen et al. 2017, 2020), but without global consensus (e.g., Silva Aguirre et al. 2018). However, the stellar thin disc component shows a larger dispersion, along with a significant scatter in the age-metallicity relation (AMR). This signature has been found to be a sign of the superposition of different stellar populations in the thin disc, with different enrichment histories and birth radii (Carlberg et al. 1985; Friel 1995; Nordström et al. 2004; Schönrich \& Binney 2009; Wojno et al. 2016).

Furthermore, the radial migration of stars in the Galaxy, through churning and blurring, is supported by theory (Sellwood \& Binney 2002; Schönrich \& Binney 2009; Di Matteo et al. 2013) and backed up by observational evidence provided by different spectroscopic stellar surveys such as RAVE (Kordopatis et al. 2015b), HARPS (Hayden et al. 2017; Minchev et al. 2018), and APOGEE (Kordopatis et al. 2017; Feltzing et al. 2020). The presence of stars with supersolar metallicity $([\mathrm{M} / \mathrm{H}] \geq+0.1 \mathrm{dex})$ and circular orbits at solar galactocentric distances has been interpreted as clear evidence of stellar migration from inner birth regions in the Galaxy. In addition, migrated stars are expected to cause a flattening of the radial abundance gradients with time (Boissier \& Prantzos 1999; Hou et al. 2000; Roškar et al. 2008; Schönrich \& Binney 2009; Pilkington et al. 2012; Minchev et al. 2018; Magrini et al. 2017; Vincenzo \& Kobayashi 2020). The temporal and spatial dimensions are therefore crucial for interpreting and clarifying the present-day chemodynamical relations in order to disentangle the formation and evolution of the Galactic disc. In this paper, we refer to radial migration as the churning process, or simply 'churning'.

In a previous analysis (Santos-Peral et al. 2020), we showed a significant improvement in the precision of $[\mathrm{Mg} / \mathrm{Fe}]$ abundance estimates by carrying out an optimisation of the spectral normalisation procedure, in particular for the metal-rich population $([\mathrm{M} / \mathrm{H}]>0)$. The followed methodology made it possible to highlight a decreasing trend in the $[\mathrm{Mg} / \mathrm{Fe}]$ abundance even at supersolar metallicites, partly solving the apparent discrepancies between the observed flat trend in the metal-rich disc (Adibekyan et al. 2012; Hayden et al. 2015, 2017; Mikolaitis et al. 2017; Buder et al. 2019) and the steeper slope predicted by chemical-evolution models (Chiappini et al. 1997; Romano et al. 2010; Spitoni et al. 2020; Palla et al. 2020). In this paper, we use these new $[\mathrm{Mg} / \mathrm{Fe}]$ abundance measurements in order to study their impact on the reported chemodynamical features (radial chemical abundance gradients, role of churning, age-abundance relations), and therefore on the interpretation of the Galactic disc evolution. We used the highspectral-resolution AMBRE:HARPS dataset (De Pascale et al. 2014), and analysed 366 main sequence turn-off (MSTO) stars in the local solar neighbourhood $(d<300 \mathrm{pc}$ from the Sun), for which we estimated ages and kinematical and dynamical parameters using the accurate astrometric measurements of the Gaia space mission.

The paper is organised as follows. In Sect. 2, we introduce the observational data sample used for the analysis. In Sect. 3, we show the radial chemical abundance gradients with Galactocentric radius and explore the effects of churning in the thin disc sample. We present our results on $[\mathrm{Mg} / \mathrm{Fe}]$ and metallicity as a function of stellar age and orbital properties in Sect. 4. In Sect. 5, we discuss the proposed scenario for the formation and evolution of the Galactic disc. We conclude with a summary in Sect. 6.

\section{Data}

\subsection{The AMBRE:HARPS sample}

The AMBRE Project, described in de Laverny et al. (2013), is a collaboration between the Observatoire de la Côte d'Azur (OCA) and the European Southern Observatory (ESO) to automatically and homogeneously parametrise archived stellar spectra from ESO spectrographs: FEROS, HARPS, and UVES. The stellar atmospheric parameters $\left(T_{\text {eff }}, \log (g),[\mathrm{M} / \mathrm{H}]\right.$, $[\alpha / \mathrm{Fe}])$ were derived by the multi-linear regression algorithm MATISSE (MATrix Inversion for Spectrum SynthEsis, Recio-Blanco et al. 2006), using the AMBRE grid of synthetic spectra (de Laverny et al. 2012). Additionally, the AMBRE Project estimates the radial velocity $\left(v_{\text {rad }}\right)$ by a cross-correlation function between the observed spectra and the used synthetic templates.

For the present paper, we only considered a subsample of the AMBRE:HARPS spectral dataset ${ }^{1}\left(R \sim 115000\right.$, described $^{2}$ in De Pascale et al. 2014) that corresponds to 494 MSTO stars in the solar neighbourhood, selected and used in Hayden et al. (2017). These latter authors made the sample selection by requiring $M_{J}<3.75$ and $3.6<\log g<4$.4. The external uncertainties (estimated by comparison with external catalogues) on $T_{\text {eff }}$, $\log (g),[\mathrm{M} / \mathrm{H}],[\alpha / \mathrm{Fe}]$, and $v_{\text {rad }}$ are $93 \mathrm{~K}, 0.26 \mathrm{~cm} \mathrm{~s}^{-2}, 0.08 \mathrm{dex}$, $0.04 \mathrm{dex}$, and $1 \mathrm{~km} \mathrm{~s}^{-1}$, respectively. Relative errors from spectra to spectra are much lower. As mentioned above, the stellar $[\mathrm{Mg} / \mathrm{Fe}]$ abundances were derived following the methodology described in Santos-Peral et al. (2020), where the spectral normalisation procedure was optimised for the different stellar types and each particular $\mathrm{Mg}$ line separately. These abundances present an overall internal error of around 0.02 dex and an average external uncertainty of 0.01 dex with respect to four identified Gaia-benchmark stars (18 Sco, HD 22879, Sun, and $\tau$ Cet) from Jofré et al. (2015).

\subsection{Gaia DR2: photometry, astrometry, and distances}

We used astrometric (Lindegren et al. 2018) and photometric data (Evans et al. 2018, full passband information for BP and RP) from the Gaia DR2 catalogue (Gaia Collaboration 2018), along with distances estimated by Bailer-Jones et al. (2018) from Gaia DR2 parallaxes using a Bayesian approach. We point out that, as the analysed sample is within a radius of $300 \mathrm{pc}$ around the Sun, the parallax uncertainties $\left(\sigma_{\varpi} / \varpi<3 \%\right.$ for our stars) and the choice of prior have little impact on the distance results. For the same reasons, new astrometric information from Gaia EDR3 will not affect our conclusions.

\footnotetext{
1 The AMBRE analysis of the HARPS spectra comprises the observations collected from October 2003 to October 2010 with the HARPS spectrograph at the $3.6 \mathrm{~m}$ telescope at the La Silla Paranal Observatory, ESO (Chile).
} 

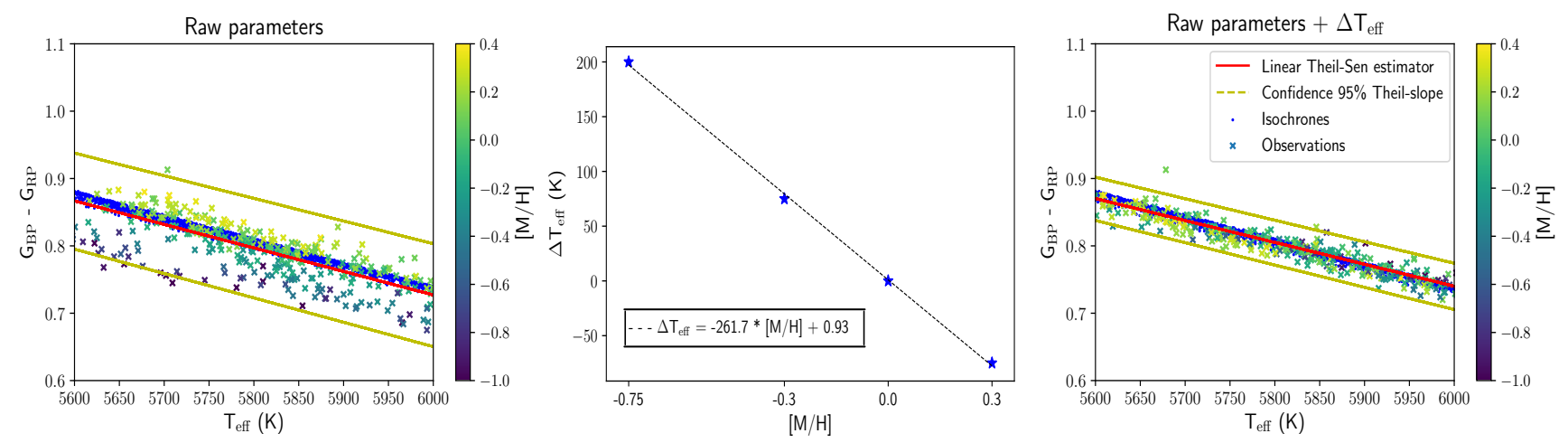

Fig. 1. Colour-temperature relation of the MSTO stars in the working sample (colour-coded according to metallicity) and the isochrone models (blue points on a straight line), for the original derived AMBRE effective temperatures (left panel) and after the offset correction in $T_{\text {eff }}($ right panel). The linear Theil-Sen estimator method (red line) was applied to the stellar parameter values. The green lines delimiter the lower and upper bound of the $95 \%$ confidence interval of the Theil-Sen linear regression method. Middle panel: observed linear $T_{\text {eff }}-$ offset $\left(\Delta T_{\text {eff }}=T_{\text {eff,MODEL }}-\right.$ $T_{\text {eff,OBSERVATION }}$ ) for different metallicity values.

We performed a cross-match of the whole AMBRE:HARPS database with the Gaia DR2 catalogue through the CDS interface, looking for a match in a radius of 5 arcsec. This allowed us to assign a Gaia ID to each spectrum, identifying the different spectra of the same star. In order to verify the goodness of the cross-match, we compared the spectroscopically derived $T_{\text {eff }}$ and radial velocities with those provided by Gaia DR2. As the number of sources with $T_{\text {eff }}$ determinations in Gaia DR2 is not very large for the AMBRE:HARPS sample, we also estimated $T_{\text {eff }}$ values from 2MASS (Skrutskie et al. 2006) and APASS (Henden et al. 2018) photometry, following González Hernández \& Bonifacio (2009) (after performing the subsequent cross-match in the same way as we did with Gaia), in order to compare with the spectroscopic ones. As an additional check, we also estimated the corresponding 2MASS and APASS photometric magnitudes from Gaia photometry. Thus, we verified the correct identification of stars in the cross-match by comparing the $T_{\text {eff }}$, the photometric magnitudes, and the radial velocities for each spectrum separately. We find that the median differences among AMBRE:HARPS, 2MASS, APASS, and Gaia data are lower than $150 \mathrm{~K}$ in $T_{\text {eff }}$, and around 0.1 for the photometric magnitudes $J, H$, and $K$. As far as the radial velocities are concerned, we were only able to compare AMBRE:HARPS and Gaia measurements, and find a median difference of about $0.02 \mathrm{~km} \mathrm{~s}^{-1}$. All these checks confirm that the AMBRE/Gaia cross-match can be used with confidence.

Finally, we selected a cleaner spectra sample by excluding those spectra whose atmospheric parameters $\left(T_{\text {eff }}, \log (g),[\mathrm{M} / \mathrm{H}]\right.$, and $[\alpha / \mathrm{Fe}])$ differ by more than two sigma from the mean value of the star. In addition, those stars with more than five observed spectra ( $\geq 5$ repeats) that present $\sigma_{\text {vrad }}>5 \mathrm{~km} \mathrm{~s}^{-1}$ were ruled out as binary system candidates. The remaining stars of the sample in the analysis present $\sigma_{\text {vrad }}<1 \mathrm{~km} \mathrm{~s}^{-1}$.

\subsection{Ages}

We restricted our sample to MSTO stars in order to estimate reliable ages using the isochrone-fitting method described in Kordopatis et al. (2016). For each individual star, we computed the age probability distribution function (PDF) by projecting the stellar parameters $\left(T_{\text {eff }}, \log g,[\mathrm{M} / \mathrm{H}]\right)$, the BP-RP colour, and the $\mathrm{G}$ absolute magnitude on the PARSEC isochrones (Bressan et al. 2012), linearly scaled in age in steps of $0.1 \mathrm{Gyr}$ from 0 to $15 \mathrm{Gyr}$, with the Evans et al. (2018) colour transformation. Every of the parameters and magnitudes were weighted by their respective error bars, and each isochrone point was weighted by mass following Zwitter et al. (2010).

We first checked that the relation between the effective temperature (derived spectroscopically) and the colour (from Gaia DR2) was on the same scale as the one used by the isochrone models. As shown in the left panel of Fig. 1, a metallicity-dependent disagreement was found. The observed offset was evaluated for four different metallicity values ${ }^{2}$, showing a linear dependence on the metallicity (middle panel):

$\Delta T_{\text {eff }}(K)=(-261.7 \pm 5.4) *[\mathrm{M} / \mathrm{H}](\mathrm{dex})+(0.93 \pm 2.32)$.

Figure 1 shows, for all the metallicities, the colourtemperature relation before (left panel) and after applying the correction in the AMBRE effective temperatures (right panel) in order to put them on the same temperature scale as the PARSEC isochrones. We determined the dispersion in the colour BP-RP differences between the observations and the models, finding an improvement from $\sigma_{\Delta(\mathrm{BP}-\mathrm{RP})} \approx 0.028$ to $\sigma_{\Delta(\mathrm{BP}-\mathrm{RP})} \approx$ 0.018 (before and after applying the $T_{\text {eff }}$-offset, respectively). We therefore used the shifted stellar temperatures (points from the right-hand panel) in the projection on the PARSEC isochrones to estimate the final stellar ages.

Moreover, the estimated distance from Gaia DR2 parallaxes was taken into account to compute the distance modulus of each star in order to calculate its absolute magnitude. We firstly estimated the Galactic extinction for our sample by implementing the 3D dust maps from Green et al. (2018), and also Schlegel et al. (1998) maps with the proposed correction by Sharma et al. (2014, see Eq. (24)) in order to not overestimate the reddening. We calculated a negligible extinction $(E(B-V) \lesssim 0.025 \mathrm{mag})$ for most of the observed stars, while some cases showed significant values but with uncertainties as high as the estimated correction. The very low derived extinctions are consistent with the fact that our sample is in the solar vicinity $(d<300 \mathrm{pc})$. As a consequence, we decided not to apply any extinction correction, as they have a negligible impact on our results. Furthermore, we did not adopt any prior either for the Galaxy model or for the age as a function of other stellar parameters such as $[\mathrm{M} / \mathrm{H}]$ or $[\mathrm{Mg} / \mathrm{Fe}]$. We assumed a uniform star formation history to avoid prioritising a particular formation epoch.

$2[\mathrm{M} / \mathrm{H}]=[-0.75,-0.3,0.0,+0.3]$. 

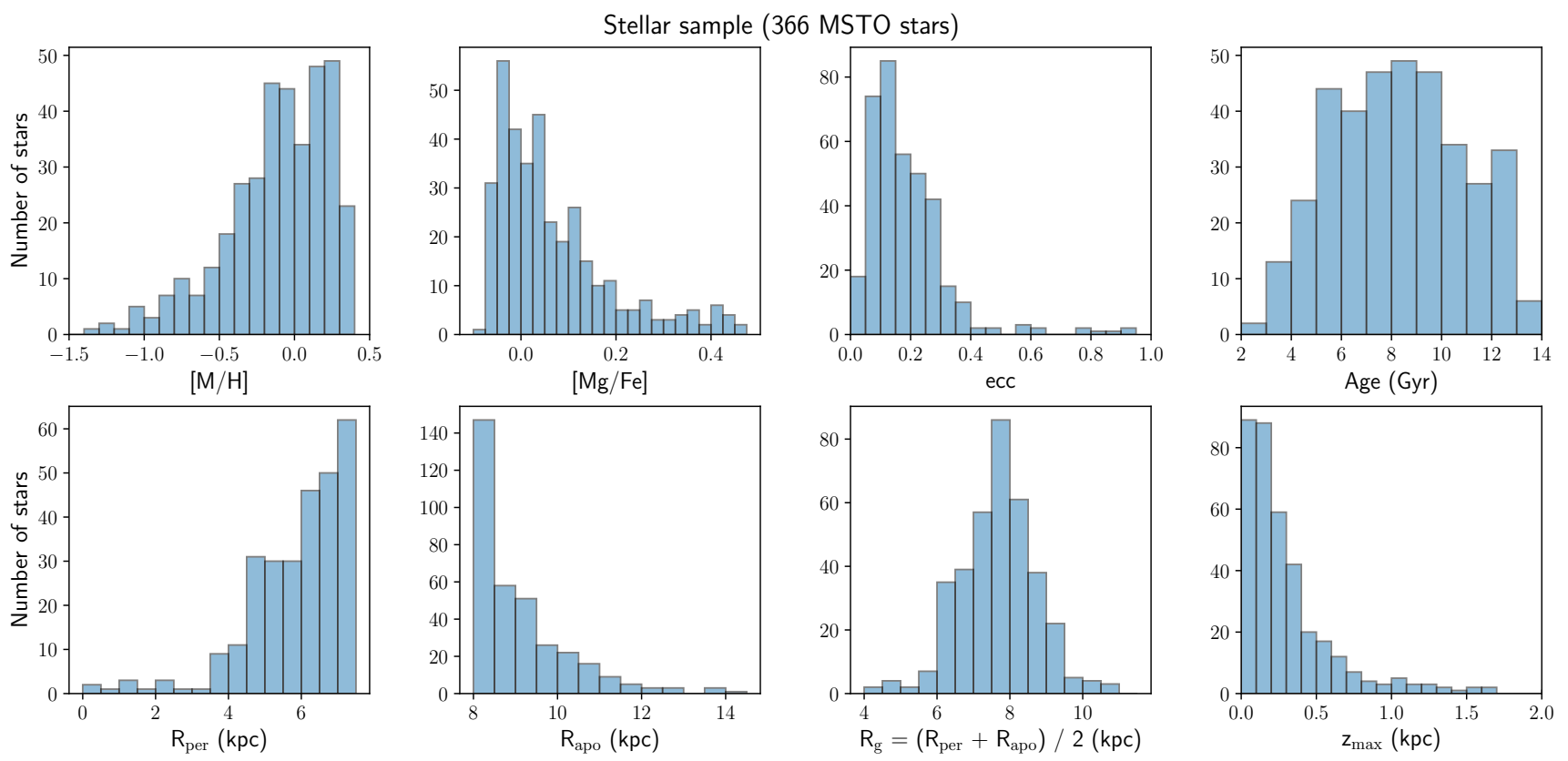

Fig. 2. Distribution of the main properties ([M/H], $[\mathrm{Mg} / \mathrm{Fe}]$, eccentricity, age, $\left.R_{\text {per }}, R_{\text {apo }}, R_{g}, z_{\max }\right)$ of the selected MSTO stars.

Finally, we excluded the stars whose mean, median, and mode age PDF values differed from one another by more than 2 Gyr, leading to a total of 366 stars with a very reliable age estimate. The adopted age for each star was the one derived by the mean of the PDF. The age distribution for our data sample ranges from 2.5 to $13.5 \mathrm{Gyr}$, with an average relative standard deviation of $\sigma \sim 20 \%$ around the mean PDF value. The absence of stars younger than 2.5 Gyr may be due to a possible bias inherent to the sample.

\subsection{Kinematic and orbital properties}

We estimated the orbital parameters with the Gaia DR2 astrometric positions and proper motions, the calculated distances by Bailer-Jones et al. (2018), and the spectroscopic radial velocities determined by the AMBRE analysis procedure (see Worley et al. 2012; De Pascale et al. 2014). For this purpose, we used the Python code galpy (Bovy 2015), together with the MWpotential2014: a Milky-Way-like gravitational potential that is the sum of a power-law density profile for the bulge (power-law exponent of -1.8 and a cut-off radius of $1.9 \mathrm{kpc}$ ), a Miyamoto-Nagai potential for the disc (Miyamoto \& Nagai 1975), and a Navarro-Frenk-White potential to model the halo (Navarro et al. 1997). The orbits were integrated over $10 \mathrm{Gyr}$ in order to evaluate the pericentres $\left(R_{\mathrm{per}}\right)$ and the apocentres $\left(R_{\mathrm{apo}}\right)$, as well as the maximum heights above the Galactic plane $\left(\left|z_{\max }\right|\right)$. As we analysed a local stellar sample in the solar neighbourhood (large parallaxes: $3<\varpi<50$ mas), the parallax bias found in the Gaia DR2 data, for example the global shift of $\delta_{\varpi}=-0.029$ mas (Lindegren et al. 2018) or $\delta_{\varpi}=-0.054$ mas by later studies (Schönrich et al. 2019; Graczyk et al. 2019), has a negligible effect on the estimated kinematic and orbital quantities.

Figure 2 shows the distribution of the main stellar properties of our final sample, consisting of 366 MSTO stars. These are Galactic disc stars, describing relatively circular prograde orbits $(0<e \lesssim 0.4)$ close to the Galactic plane $\left(\left|z_{\max }\right| \lesssim 1 \mathrm{kpc}\right)$, and are evenly distributed in age from $\sim 2.5$ to $13.5 \mathrm{Gyr}$. We find a significant fraction of stars that seem to be passing through our local limited sample on the present day, with $R_{\text {per }}$ values lower than $6 \mathrm{kpc}$, and also some of them reaching $R_{\text {apo }}$ values of greater than $10 \mathrm{kpc}$ from the Galactic centre. We used the guiding centre radius $\left(R_{g}\right)$ as an estimate of the current value of the Galactocentric radius $\left(R_{\mathrm{GC}}\right)$, because it is a useful tracer of the radial migration of the different stellar populations in the Galactic disc (e.g., Binney 2007; Kordopatis et al. 2017). For this work, $R_{g}$ was calculated as the average between the pericentre and the apocentre of the orbit of the star: $R_{g}=\left(R_{\text {per }}+R_{\text {apo }}\right) / 2$. The sample is well distributed in Galactocentric $R_{g}$ from 4 to $11 \mathrm{kpc}$.

In order to estimate the error in $R_{g}$, we re-calculated the orbits using the upper and lower distance limit derived by Bailer-Jones et al. (2018). The comparison shows a negligible uncertainty of less than 1 parsec. We also calculated the orbits using other potentials: the MWpotential2014 adding a bar (DehnenBarPotential) on the one hand, and the potential presented by McMillan (2017) on the other. For both cases, the differences in the $R_{g}$ value were around $0.2 \mathrm{kpc}$ with respect to the initial calculated ones. Our results are robust against the choice of the Galactic potential model and the uncertainty in the parameters.

\section{Radial chemical trends and stellar migration}

In this section, we first present an exploration of the present-day distribution of $[\mathrm{Mg} / \mathrm{Fe}]$ and $[\mathrm{M} / \mathrm{H}]$ in the thin disc as a function of Galactocentric position and age. We then present our analysis of the impact of radial migration in the solar neighbourhood.

\subsection{Definition of the thin disc}

Here we adopt a chemical definition of the thin disc, based on the $[\mathrm{Mg} / \mathrm{Fe}]$ content in a given $[\mathrm{M} / \mathrm{H}]$ bin.

Figure 3 illustrates the chemical separation in the $[\mathrm{Mg} / \mathrm{Fe}]-$ $[\mathrm{M} / \mathrm{H}]$ plane for our working sample, classifying the stars into high- and low- $[\mathrm{Mg} / \mathrm{Fe}]$ sequences. First of all, we selected stars older than 12 Gyr. According to previous works in the literature 


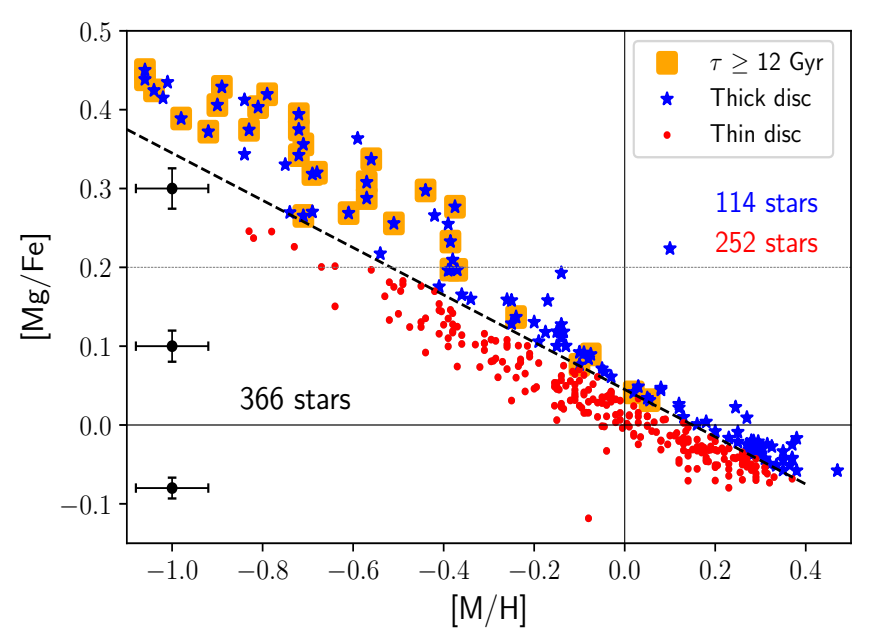

Fig. 3. $[\mathrm{Mg} / \mathrm{Fe}]$ vs. $[\mathrm{M} / \mathrm{H}]$ for our working sample. The stars with ages older than $12 \mathrm{Gyr}$ are highlighted with orange squares. The black dashed line defines the thin(red circles)-thick(blue stars) disc chemical separation. The mean estimated errors are represented on the left-hand side for three different intervals in $[\mathrm{Mg} / \mathrm{Fe}]$.

(cf. Fuhrmann 2011; Haywood et al. 2013; Hayden et al. 2017), this would trace the old (thick) disc population. These stars, highlighted by orange squares in Fig. 3, are mostly $[\mathrm{Mg} / \mathrm{Fe}]-$ enhanced $([\mathrm{Mg} / \mathrm{Fe}] \gtrsim+0.2$ dex $)$, but span a wide range in $[\mathrm{M} / \mathrm{H}]$, reaching the solar values. We used the lower $[\mathrm{Mg} / \mathrm{Fe}]$-bound $\mathrm{fit}^{3}$ to these stars to chemically define the thick (blue stars in Fig. 3) and the thin disc (red points in Fig. 3). This line clearly separates the two chemical sequences at low metallicities, yet the extrapolation to the metal-rich tail $([\mathrm{M} / \mathrm{H}]>-0.3 \mathrm{dex})$ is rather arbitrary and the classified thick disc, also called the $\alpha$-rich metal-rich population in previous works (e.g., Adibekyan et al. 2012), has an ad hoc assignment that needs to be analysed in detail.

\section{Distinct metal-rich populations?}

The difficulty in separating the thin and thick disc stars in terms of chemical abundances at high metallicities is a matter of debate in the literature. On the one hand, Adibekyan et al. (2012) and Mikolaitis et al. (2017) found a gap in metallicity $([\mathrm{Fe} / \mathrm{H}] \approx$ -0.2 dex) for the thick disc population in a sample of dwarf stars from HARPS data. These authors used this gap to chemically define the thick disc and the $\alpha$-rich metal-rich sequences separately. Adibekyan et al. (2012) showed that high- $\alpha$ metal-rich stars were on average older than thin disc stars, but with similar kinematics and orbits to the thin disc population. On the other hand, Hayden et al. (2015) and Buder et al. (2019) (for two independent samples of giants from APOGEE and dwarfs from GALAH DR2 data, respectively) found a continuous evolution for the thick disc sequence, describing an independent track from the thin disc up to supersolar metallicities.

Moreover, due to possible ignored uncertainties in our abundance estimates (Santos-Peral et al. 2020), each sample is expected to contain a fraction of contamination from the other sample in the metal-rich regime $([\mathrm{M} / \mathrm{H}]>-0.3 \mathrm{dex})$. To evaluate the advantages of a separate treatment of the thick disc metalrich population with respect to the thin disc one, we assessed the possible differences between the classified high- and low$[\mathrm{Mg} / \mathrm{Fe}]$ disc sequence populations in the metal-rich regime.

\footnotetext{
$[\mathrm{Mg} / \mathrm{Fe}]=-0.3 \cdot[\mathrm{M} / \mathrm{H}]+0.045(\mathrm{dex})$.
}

Figure 4 shows the chemodynamical properties of the high(blue) and low- $[\mathrm{Mg} / \mathrm{Fe}]$ (brown) disc metal-rich stars $([\mathrm{M} / \mathrm{H}]>$ $-0.3 \mathrm{dex})$. Some of the high- $[\mathrm{Mg} / \mathrm{Fe}]$ disc metal-rich stars show low eccentric orbits, young ages, and are close to the plane (low$\left.z_{\max }\right)$, similarly to the metal-rich low- $[\mathrm{Mg} / \mathrm{Fe}]$ population. The Kolmogorov-Smirnov test between the two samples does not allow us to reject that these high metallicity stars are truly different stellar populations. This is particularly true for the $z_{\max }$ and the eccentricity distributions, while the $p$-value is smaller for the radial and age distribution.

For comparison, Fig. 5 illustrates the same analysis for the metal-poor stellar subsample $([\mathrm{M} / \mathrm{H}] \leq-0.3 \mathrm{dex})$. Besides the clear $[\mathrm{Mg} / \mathrm{Fe}]$ distinction observed in Fig. 3 at this metallicity regime, it seems reasonable to interpret the reported $p$-values as evidence in favour of two different stellar populations ( $p$-values $<10^{-3}$ for the orbital parameter distributions: eccentricity, $R_{\text {per }}, R_{\text {apo }}$, and $R_{g}$ ). The high-[Mg/Fe] metal-poor population clearly shows a more centrally concentrated distribution (reaching the innermost regions: $R_{\mathrm{per}} \sim 0-7 \mathrm{kpc}$ and $R_{g}$ down to $4 \mathrm{kpc}$ from the Galactic centre) and presents a higher eccentricity tail. On the contrary, the low- $[\mathrm{Mg} / \mathrm{Fe}]$ metal-poor population reaches the outer parts of the Galactic disc (up to $R_{\text {apo }} \sim 14 \mathrm{kpc}$ and $\left.R_{g} \sim 10 \mathrm{kpc}\right)$ and only describes circular orbits $(e \lesssim 0.3)$.

In conclusion, we only decided to apply the chemical criterion described in Fig. 3 in the metal-poor regime $([\mathrm{M} / \mathrm{H}] \leq$ -0.3 dex) in order to minimise the thick disc contamination in the study of the thin disc properties. Nevertheless, the similarities observed in the metal-rich regime $([\mathrm{M} / \mathrm{H}]>-0.3 \mathrm{dex})$ do not support a thin-thick disc separation at high metallicities. As a consequence, we consider all the metal-rich stars as part of the thin disc population in the following gradient and radial migration study, and a global consideration of the entire disc population is adopted for the disc evolution analysis in Sect. 4.

\subsection{Present-day chemical abundance gradients}

Table 1 shows the radial gradients of $[\mathrm{M} / \mathrm{H}]$ and $[\mathrm{Mg} / \mathrm{Fe}]$ for the thin disc stars, assuming $R_{g}$ as their true position in the Galaxy. The values of the slope and the uncertainties are reported in Table 1, and come from a Theil-Sen regression model.

We find a negative gradient of $-0.099 \pm 0.031 \mathrm{dex} \mathrm{kpc}^{-1}$ for $[\mathrm{M} / \mathrm{H}]$ and a positive gradient of $+0.023 \pm 0.009 \mathrm{dex} \mathrm{kpc}^{-1}$ for the $[\mathrm{Mg} / \mathrm{Fe}]$ abundance. Both chemical gradients are flatter for young stars $(\leq 6 \mathrm{Gyr})$, although the differences are within the slope uncertainties. In contrast, for the same stellar subsample, the Mikolaitis et al. (2017) $[\mathrm{Mg} / \mathrm{Fe}]$ abundances lead to a shallower gradient: $+0.004 \pm 0.007 \mathrm{dex} \mathrm{kpc}^{-1}$.

The $[\mathrm{Mg} / \mathrm{Fe}]$ abundances from Santos-Peral et al. (2020), obtained with a careful treatment of the spectral continuum placement, show a decreasing trend in the $[\mathrm{Mg} / \mathrm{Fe}]$ abundance even at supersolar metallicites. In contrast, previous observational studies of the solar neighbourhood observed a flattened trend (e.g., Adibekyan et al. 2012; Mikolaitis et al. 2017). As a consequence, it seems that the reported improvement in the $[\mathrm{Mg} / \mathrm{Fe}]$ abundance precision for the metal-rich disc implies a significant change in the radial gradient measurement, showing steeper slopes. As an additional test, we also explored the possible influence of a thin-thick disc misclassification (see Sect. 3.1) by not including in our thin disc radial gradient the measurements of the high- $[\mathrm{Mg} / \mathrm{Fe}]$ metal-rich stars, finding similar results: $d[\mathrm{Mg} / \mathrm{Fe}] / d R_{g}=+0.025 \pm 0.009 \mathrm{dex} \mathrm{kpc}{ }^{-1}$ (whole sample), $+0.020 \pm 0.015 \mathrm{dex} \mathrm{kpc}^{-1}$ (young stars), and $+0.028 \pm 0.010 \mathrm{dex} \mathrm{kpc}^{-1}$ (old stars). Therefore, the observed steeper $[\mathrm{Mg} / \mathrm{Fe}]$ gradient is not affected by the possible 

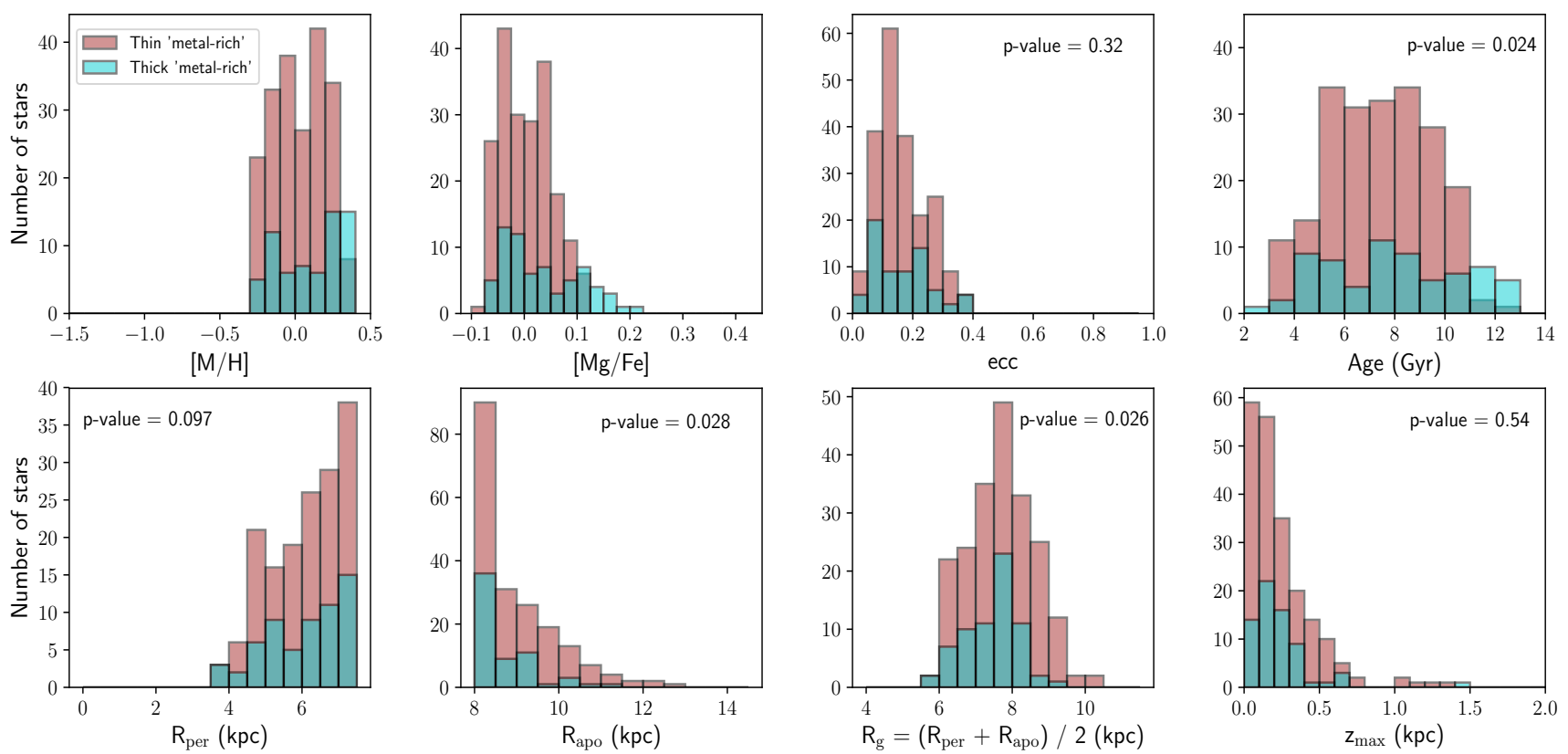

Fig. 4. Same stellar properties as Fig. 2, separated into the chemically defined thin (brown) and thick (blue) disc stellar populations for the metalrich subsample $([\mathrm{M} / \mathrm{H}]>-0.3 \mathrm{dex})$. The $p$-values of the two-sample Kolmogorov-Smirnov tests are reported for each parameter distribution.
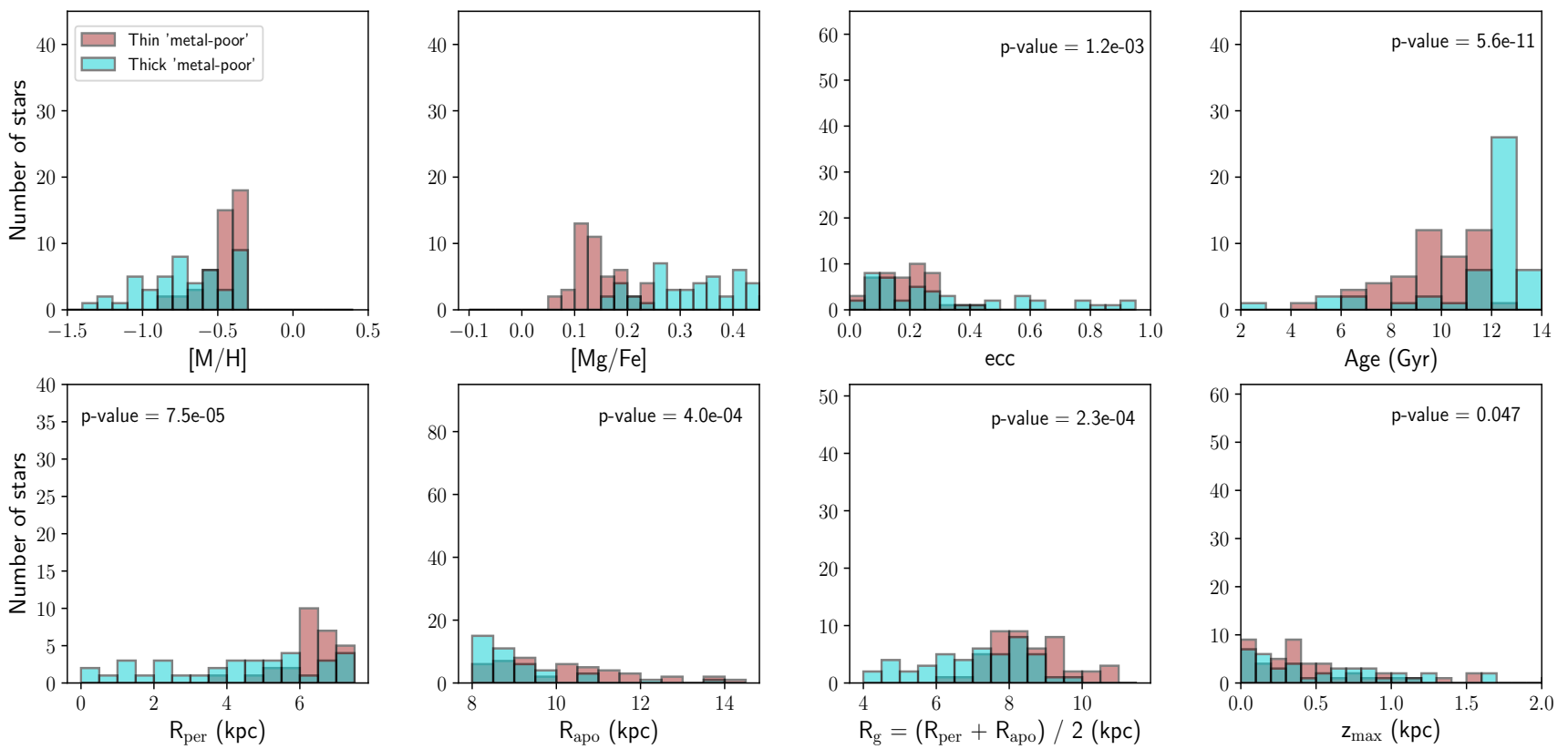

Fig. 5. Same as Fig. 4 but for the metal-poor subsample $([\mathrm{M} / \mathrm{H}] \leq-0.3$ dex $)$, with the respective $p$-values of the two-sample Kolmogorov-Smirnov tests.

thin-thick disc contamination, and seems to be a direct result of the newly derived $[\mathrm{Mg} / \mathrm{Fe}]$ abundances.

\section{Comparison with literature studies}

The radial metallicity gradient value in the Galactic disc, along with its evolution with time, are still debated in the literature, with negative slopes ranging from -0.04 to $-0.1 \mathrm{dex} \mathrm{kpc}^{-1}$ for the analysis of different Galactic populations: planetary nebulae (PNe), HII regions, open clusters, variable (Cepheids) and field stars. Our measured value is therefore in agreement within the literature range (see Table 2). It is worth noting that the gradients shown here correspond to works with different target selections and implemented methodologies to estimate abundances and distances. Depending on the work, the metallicity value was measured globally $([\mathrm{M} / \mathrm{H}])$ or considering only iron lines $([\mathrm{Fe} / \mathrm{H}])$, and the radial distance estimate comes from either the guiding centre radius of the stellar orbit $\left(R_{g}\right)$ or the Galactocentric radius of the $\operatorname{star}\left(R_{\mathrm{GC}}\right)$.

First, Table 2 shows how our metallicity gradient estimation is qualitatively in good agreement with previous analyses of field stars from different Galactic surveys. Compatible results were obtained using data from SEGUE $(R \sim 2000$, Cheng et al. 2012), RAVE $(R \sim 7500$, Boeche et al. 2013a), APOGEE 
Table 1. Radial abundance gradients $\left(\mathrm{dex} \mathrm{kpc}^{-1}\right)$ in the range $6 \leq R_{g} \leq 11 \mathrm{kpc}$ for Galactic thin disc stars.

\begin{tabular}{lccc}
\hline \hline & $\frac{d[\mathrm{M} / \mathrm{H}]}{d R g}$ & $\frac{d[\mathrm{Mg} / \mathrm{Fe}]}{d R g}$ & $\frac{d[\mathrm{Mg} / \mathrm{Fe}]}{d R g}$ \\
\hline Whole sample & $-0.099 \pm 0.031$ & $+0.023 \pm 0.009$ & $+0.004 \pm 0.007$ \\
Young ( $\leq 6 \mathrm{Gyr})$ & $-0.088 \pm 0.063$ & $+0.021 \pm 0.015$ & $-0.007 \pm 0.011$ \\
Old (> 6 Gyr) & $-0.106 \pm 0.035$ & $+0.025 \pm 0.010$ & $+0.010 \pm 0.007$ \\
\hline
\end{tabular}

Notes. ${ }^{(\dagger)}$ Abundances from Mikolaitis et al. (2017).

Table 2. Radial abundance gradients $\left(\mathrm{dex} \mathrm{kpc}^{-1}\right)$ in the Galactic thin disc from the literature.

\begin{tabular}{|c|c|c|c|c|c|}
\hline Abundance & Value & Work & Tracer & Thin disc definition & Database \\
\hline$\frac{d[\mathrm{Fe} / \mathrm{H}]}{d R_{\mathrm{GC}}}$ & $-0.066 \pm 0.037$ & Cheng et al. (2012) & Field dwarf stars & $\begin{aligned} 0.15<|z| & <0.25 \mathrm{kpc}\left(6<R_{\mathrm{GC}}<16 \mathrm{kpc}\right) \\
& \left(6<R_{\mathrm{GC}}<16 \mathrm{kpc}\right)\end{aligned}$ & SEGUE \\
\hline$\frac{d[\mathrm{Fe} / \mathrm{H}]}{d R_{g}}$ & $-0.065 \pm 0.003$ & Boeche et al. (2013a) & Field dwarf stars & $\begin{array}{c}\left|z_{\max }\right|<0.4 \mathrm{kpc} \\
\left(4.5<R_{q}<9.5 \mathrm{kpc}\right)\end{array}$ & RAVE \\
\hline$\frac{d[\mathrm{M} / \mathrm{H}]}{d R_{\mathrm{GC}}}$ & $-0.090 \pm 0.002$ & Hayden et al. (2014) & Field giant stars & $\begin{array}{c}\text { Chemical }+|z|<0.25 \mathrm{kpc} \\
\left(5<R_{\mathrm{GC}}<15 \mathrm{kpc}\right)\end{array}$ & APOGEE \\
\hline$\frac{d[\mathrm{Fe} / \mathrm{H}]}{d R_{g}}$ & $-0.074 \pm 0.010$ & Anders et al. (2014) & Field giant stars & $\begin{array}{c}\left|z_{\max }\right|<0.4 \mathrm{kpc} \\
\left(6<R_{q}<11 \mathrm{kpc}\right)\end{array}$ & APOGEE Gold \\
\hline$\frac{d[\mathrm{Fe} / \mathrm{H}]}{d R_{\mathrm{GC}}}$ & $-0.068 \pm 0.016$ & Bergemann et al. (2014) & Field stars & $\begin{array}{c}|z|<0.3 \mathrm{kpc} \\
\left(6<R_{\mathrm{GC}}<9.5 \mathrm{kpc}\right)\end{array}$ & GES UVES \\
\hline$\frac{d[\mathrm{Fe} / \mathrm{H}]}{d R_{\mathrm{GC}}}$ & $-0.045 \pm 0.012$ & Mikolaitis et al. (2014) & Field stars & $\begin{array}{l}\text { Chemical }+|z|<0.608 \mathrm{kpc} \\
\quad\left(4<R_{\mathrm{GC}}<12 \mathrm{kpc}\right)\end{array}$ & GES GIRAFFE \\
\hline$\frac{d[\mathrm{Fe} / \mathrm{H}]}{d R_{\mathrm{GC}}}$ & $-0.058 \pm 0.008$ & Recio-Blanco et al. (2014) & Field stars & $\begin{array}{l}\text { Chemical }+|z|<0.7 \mathrm{kpc} \\
\left(5<R_{\mathrm{GC}}<11 \mathrm{kpc}\right)\end{array}$ & GES GIRAFFE \\
\hline$\frac{d[\mathrm{Fe} / \mathrm{H}]}{d R_{\mathrm{GC}}}$ & $\begin{array}{l}-0.053 \pm 0.029 \\
-0.094 \pm 0.008 \\
-0.091 \pm 0.060\end{array}$ & Magrini et al. (2009) & $\begin{array}{c}\leq 0.8 \mathrm{Gyr} \\
\text { OCs: } 0.8-4 \mathrm{Gyr} \\
\text { 4-11 Gyr }\end{array}$ & $\left(7<R_{\mathrm{GC}}<12 \mathrm{kpc}\right)$ & \\
\hline$\frac{d[\mathrm{Fe} / \mathrm{H}]}{d R_{G_{G}}}$ & $-0.090 \pm 0.030$ & Frinchaboy et al. (2013) & OCs & $\left(7.9<R_{\mathrm{GC}}<14 \mathrm{kpc}\right)$ & OCCAM-APOGEE \\
\hline$\frac{d[\mathrm{Fe} G / \mathrm{H}]}{d R_{\mathrm{GC}}}$ & $-0.068 \pm 0.004$ & Donor et al. (2020) & OCs & $\left(6<R_{\mathrm{GC}}<13.9 \mathrm{kpc}\right)$ & OCCAM-APOGEE \\
\hline$\frac{d[\mathrm{Fe} / \mathrm{H}]}{d R_{\mathrm{GC}}}$ & $-0.060 \pm 0.002$ & Genovali et al. (2014) & Cepheids & $\left(5<R_{\mathrm{GC}}<19 \mathrm{kpc}\right)$ & \\
\hline$\frac{d[\mathrm{Mg} / \mathrm{Fe}]}{d R_{g}}$ & $-0.009 \pm 0.002$ & Boeche et al. (2013a) & Field dwarf stars & $\begin{array}{c}\left|z_{\max }\right|<0.4 \mathrm{kpc} \\
\left(4.5<R_{g}<9.5 \mathrm{kpc}\right)\end{array}$ & RAVE \\
\hline$\frac{d[\mathrm{Mg} / \mathrm{Fe}]}{d R_{\mathrm{GC}}}$ & $+0.021 \pm 0.016$ & Bergemann et al. (2014) & Field stars & $\begin{array}{c}|z|<0.3 \mathrm{kpc} \\
\left(6<R_{\mathrm{GC}}<9.5 \mathrm{kpc}\right)\end{array}$ & GES UVES \\
\hline$\frac{d[\mathrm{Mg} / \mathrm{M}]}{d R_{\mathrm{GC}}}$ & $+0.009 \pm 0.003$ & Mikolaitis et al. (2014) & Field stars & $\begin{array}{l}\text { Chemical }+|z|<0.608 \mathrm{kpc} \\
\quad\left(4<R_{\mathrm{GC}}<12 \mathrm{kpc}\right)\end{array}$ & GES GIRAFFE \\
\hline$\frac{d[\mathrm{Mg} / \mathrm{Fe}]}{d R_{\mathrm{GC}}}$ & $+0.012 \pm 0.002$ & Recio-Blanco et al. (2014) & Field stars & $\begin{array}{l}\text { Chemical }+|z|<0.7 \mathrm{kpc} \\
\quad\left(5<R_{\mathrm{GC}}<11 \mathrm{kpc}\right)\end{array}$ & GES GIRAFFE \\
\hline$\frac{d[\mathrm{Mg} / \mathrm{Fe}]}{d R_{\mathrm{GC}}}$ & $+0.013 \pm 0.006$ & Mikolaitis et al. (2019) & Field dwarf stars & $\begin{array}{c}\text { Kinematical } \\
\left(6<R_{\mathrm{GC}}<10 \mathrm{kpc}\right)\end{array}$ & VUES \\
\hline$\frac{d[\mathrm{Mg} / \mathrm{Fe}]}{d R_{\mathrm{GC}}}$ & $+0.013 \pm 0.003$ & Genovali et al. (2015) & Cepheids & $\left(4.1<R_{\mathrm{GC}}<18.4 \mathrm{kpc}\right)$ & UVES \\
\hline$\frac{d[\mathrm{Mg} / \mathrm{Fe}]}{d R_{\mathrm{GC}}}$ & $+0.009 \pm 0.001$ & Donor et al. (2020) & OCs & $\left(6<R_{\mathrm{GC}}<13.9 \mathrm{kpc}\right)$ & OCCAM-APOGEE \\
\hline
\end{tabular}

( $R \sim$ 22 500, Hayden et al. 2014; Anders et al. 2014), and the Gaia-ESO Survey (GES) GIRAFFE $(R \sim 20000$, Mikolaitis et al. 2014; Recio-Blanco et al. 2014) and UVES data ( $R \sim 47$ 000, Bergemann et al. 2014).

Additionally, stellar open clusters (OCs) are considered a unique tool with which to study the time evolution of the radial metallicity gradients, because of their accurate derived ages and Galactocentric distances, covering wide ranges along the Galactic disc (cf. Magrini et al. 2009, OCs ages from $\sim 30 \mathrm{Myr}$ to $11 \mathrm{Gyr}$ ). Our measurement shows good agreement with the one presented by Magrini et al. (2009) for OCs older than $0.8 \mathrm{Gyr}$, and also with the overall gradient found by Frinchaboy et al. (2013) from the OCCAM survey using APOGEE data, which was recently updated by Donor et al. (2020).
Furthermore, Cepheid variable stars provide the present-day abundance gradients of the Galactic disc because they are massive stars younger than $300 \mathrm{Myr}$, and are commonly used as good distance indicators and as chemical tracers of the interstellar medium (ISM) abundance (cf. Genovali et al. 2014, and references therein). For a homogeneous sample of Galactic Cepheids observed at high spectral resolution $(R \sim 38000)$, Genovali et al. (2014) derived a consistent estimate for the present-day ISM abundance gradient.

On the other hand, the observed positive gradient in the $[\mathrm{Mg} / \mathrm{Fe}]$ abundance with radius is in close agreement with the results from Bergemann et al. (2014). However, shallower positive gradients in $[\mathrm{Mg} / \mathrm{Fe}]$ are commonly reported within the literature (e.g., Mikolaitis et al. 2014; Recio-Blanco et al. 2014; 
Mikolaitis et al. 2019, a recent analysis using the high-resolution VUES spectrograph, $R \sim 60000$ ), even finding negative values (Boeche et al. 2013a). Additionally, Genovali et al. (2015) and Donor et al. (2020) reported a similar slope resulting from an analysis of Galactic Cepheids and open clusters, respectively, over the Galactic disc.

As a consequence, our radial $[\mathrm{Mg} / \mathrm{Fe}]$ gradient is not only steeper than the one estimated from Mikolaitis et al. (2017) abundances (see Table 1), but is also steeper than other previous measurements in the literature. Once again, this is a major consequence of the Santos-Peral et al. (2020) improvement of the data for metal-rich stars. However, we point out that Perdigon et al. (2021) report a flatter radial gradient for sulphur (another $\alpha$ element) equal to $+0.012 \mathrm{dex} \mathrm{kpc}^{-1}$ for AMBRE stars and adopting a similar methodology to ours.

\subsection{Radial migration}

The stars in the Galactic disc are very likely to be scattered by resonances with the spiral arms or by giant molecular clouds that could increase the radial oscillation amplitudes around their $R_{g}$ (blurring), or change the angular momentum of the orbit (churning) (Lynden-Bell \& Kalnajs 1972; Lacey 1984; Grenon 1989, 1999; Sellwood \& Binney 2002; Schönrich \& Binney 2009; Minchev et al. 2014; Kordopatis et al. 2015b). On the one hand, stars with more eccentric and inclined orbits can belong to Galactic regions far from the Sun but reach the solar neighbourhood via blurring. However, as shown in Fig. 2, 50\% of the stars of our selected sample are on very circular orbits with $e<0.15$. Therefore, the observed stars are either expected to have been born locally or to have increased (decreased) their angular momentum via churning, increasing (decreasing) their guiding centre radius $\left(R_{g}\right)$ without changing their orbit eccentricity.

The impact of radial migration over a whole observed stellar sample is a complex, unsolved problem. In particular, its final effect on the chemical evolution and the metallicity distribution function in the solar vicinity is very uncertain, and has been suggested to be negligible (Spitoni et al. 2015; Halle et al. 2018; Vincenzo \& Kobayashi 2020; Khoperskov et al. 2021). The unique observational constraint consists in the presence of too metal-rich stars in the solar annulus that cannot be justified by chemical evolution models without an exchange of matter between different radial annuli in the Galactic disc (Grisoni et al. 2017). Under the assumption that a negative radial metallicity gradient has been present in the Galaxy since the thin disc formation epoch (Roškar et al. 2008; Magrini et al. 2009; Schönrich \& McMillan 2017; Minchev et al. 2018), and given a present-day ISM abundance in the solar vicinity of around $[\mathrm{M} / \mathrm{H}] \sim 0.0$ dex, the observed super-metal-rich stars (SMR; $[\mathrm{M} / \mathrm{H}] \gtrsim+0.1 \mathrm{dex}$ ) should have been formed in the inner disc regions (Minchev et al. 2013; Kordopatis et al. 2015b; Hayden et al. 2020).

These assumptions have been taken into account in the following analysis, although some uncertainties remain. For instance, the metallicity of forming stars in the solar vicinity is still a matter of debate, with some recent studies questioning its solar nature (e.g., open cluster analysis by Baratella et al. 2020; Spina et al. 2021), and also Delgado Mena et al. (2019) found $[\mathrm{Fe} / \mathrm{H}]>0$ (with a mean near $0.1 \mathrm{dex}$ ) for field stars younger than $1 \mathrm{Gyr}$. In addition, based on the observed cosmic dispersion in metallicity in the local Universe $(\sim 0.05$ dex, e.g., Mannucci et al. 2010) and the observational error of our measurements (0.04 dex; see Sect. 2.1), the presence of stars with

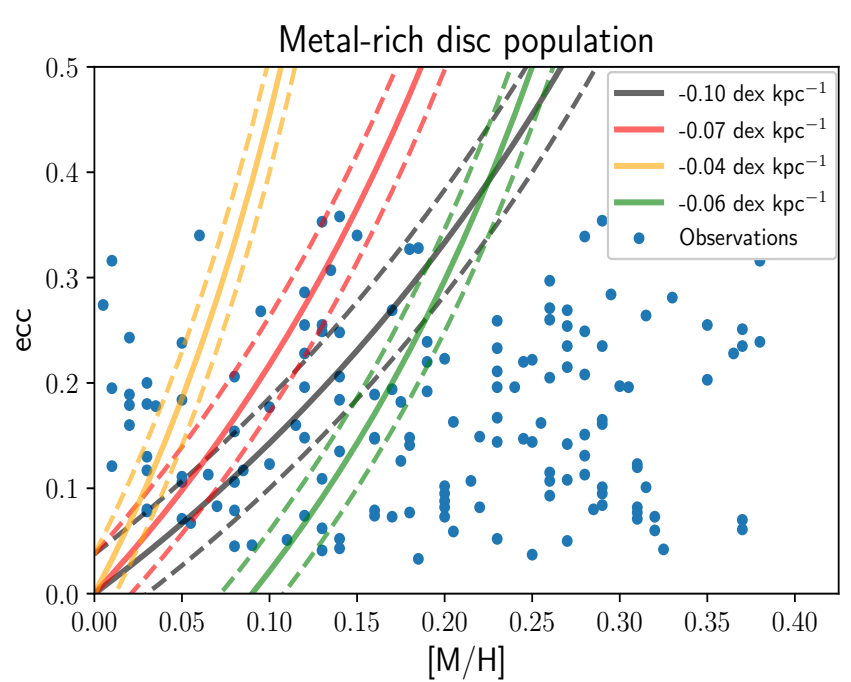

Fig. 6. Distribution of the orbital eccentricities as a function of $[\mathrm{M} / \mathrm{H}]$ for the thin disc metal-rich sample. The solid (dashed) lines indicate the required eccentricity to reach $R=8 \mathrm{kpc}( \pm 300 \mathrm{pc})$ without the need for churning (see Eq. (2)). For a fixed zero point, $\operatorname{ISM}_{[\mathrm{M} / \mathrm{H}]}\left(R_{\odot}\right)=0.0$, we studied three different ISM metallicity gradients (black, red, and orange). The green curve corresponds to the gradient from Genovali et al. (2014). The stars to the right require churning to reach the solar neighbourhood.

metallicity in the range $0.1-0.2$ could also be compatible with a chemical evolution with no radial migration in the solar annulus.

Following the procedure described in Hayden et al. (2020), we estimated the minimum required eccentricity of a star at a given metallicity to reach the solar neighbourhood exclusively due to blurring. For this purpose, we assumed the apocentre of the orbit to be the measured present-day position. Additionally, assuming a given radial ISM metallicity gradient and fixing the local ISM abundance to $\left(R_{\odot},[\mathrm{M} / \mathrm{H}]\right)=(8.0 \mathrm{kpc}, 0.0 \mathrm{dex})$, it is possible to estimate the birth radius of the star from its observed present-day $[\mathrm{M} / \mathrm{H}]$. In this framework, we selected the churned candidates through the following required minimum eccentricity relation:

$\operatorname{ecc}^{\star}([\mathrm{M} / \mathrm{H}]) \geq \frac{R}{R_{\text {birth }}([\mathrm{M} / \mathrm{H}])}-1$.

In this relation, $R$ is the present-day star position, which is assumed to lie between 7.7 and $8.3 \mathrm{kpc}$ because our sample is located within $300 \mathrm{pc}$ of the Sun, and $R_{\text {birth }}$ is the estimated birth radius of the star given its metallicity $[\mathrm{M} / \mathrm{H}]$, but also assuming an ISM gradient.

The evolution with time of the ISM radial metallicity gradient is also debated in the literature for Galactic chemical evolution models and cosmological simulations: some models predict a time invariant gradient (e.g., Magrini et al. 2009; Gibson et al. 2013), while others predict a steepening of the ISM metallicity gradient with time (e.g., Chiappini et al. 2001; Schönrich \& McMillan 2017), or a flattening with time (e.g., Boissier \& Prantzos 1999; Hou et al. 2000; Roškar et al. 2008; Pilkington et al. 2012; Minchev et al. 2018). For that reason, we decided to consider different ISM gradient values in the $R_{\text {birth }}$ estimate for the SMR stars, and study the influence of these assumptions on the conclusions.

Figure 6 shows the orbital eccentricities as a function of $[\mathrm{M} / \mathrm{H}]$ for the metal-rich disc sample. The solid lines correspond to the required eccentricity (see Eq. (2)) 

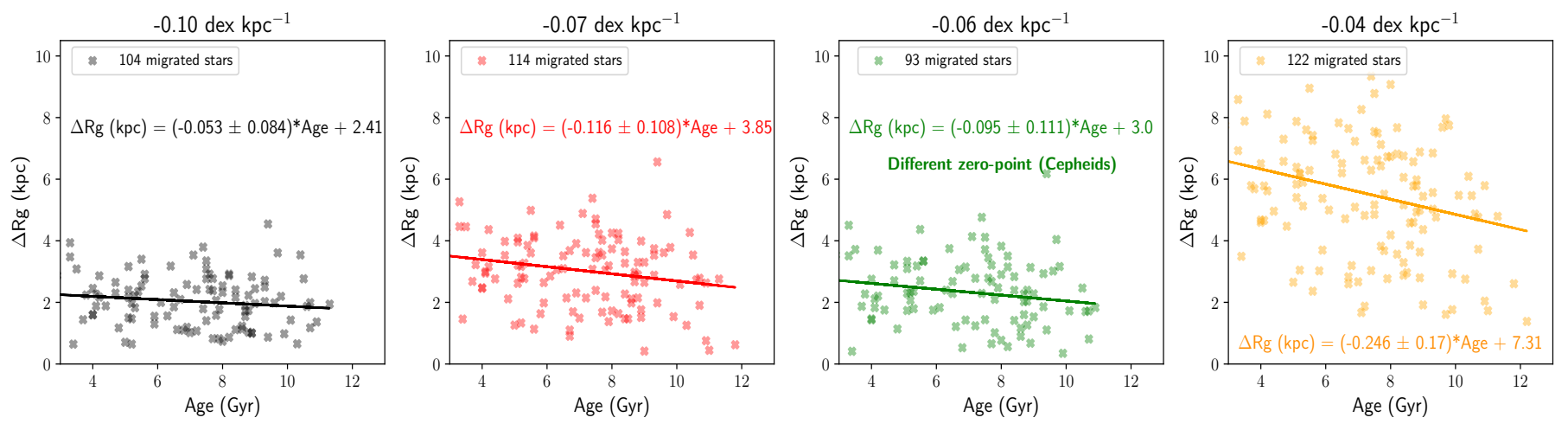

Fig. 7. Estimated covered distance $\Delta R_{g}\left(R_{g}-R_{\text {birth }}\right)$ vs. stellar age for the selected churned SMR stars ([M/H] $\left.>0.1\right)$ for different ISM metallicity gradients (see Fig. 6 and titles of the plots).

for different values of ISM radial metallicity gradients: $-0.10 \mathrm{dex} \mathrm{kpc}^{-1}$ (black), $-0.07 \mathrm{dex} \mathrm{kpc}^{-1}$ (our measured gradient for young stars in Table 1; see also Minchev et al. 2018, red), $-0.04 \mathrm{dex} \mathrm{kpc}^{-1}$ (orange), and $-0.06 \mathrm{dex} \mathrm{kpc}^{-1}$ (Cepheids analysis from Genovali et al. 2014, green). For the three first cases, we assumed $\operatorname{ISM}_{[\mathrm{M} / \mathrm{H}]}\left(R_{\odot}\right)=0.0$ to estimate $R_{\text {birth }}$ from the stellar metallicity. However, Genovali et al. (2014) have their own zero point, defined as: $[\mathrm{Fe} / \mathrm{H}]=-0.06 * R_{g}+0.57$, with a clear shift in the relation compared to the other ones assumed in this work. The impact of the ISM gradient value and the zero-point assumption on the derived $R_{\text {birth }}$, and therefore on the required eccentricity to reach the solar vicinity without the need for churning, is clearly observed. As described in Hayden et al. (2020), given the measured $[\mathrm{M} / \mathrm{H}]$ and eccentricity, stars lying to the left are able to reach the solar neighbourhood through blurring, while the stars to the right of the line are possible candidates to have migrated through churning. This is the case for most of the SMR stars $(70 \%$ of the SMR stars lie below the line that corresponds to the Cepheids analysis); they are therefore likely to have been brought to the solar neighbourhood by churning, which is in close agreement with previous studies (e.g., Kordopatis et al. 2015a; Wojno et al. 2016). However, it is worth noting that the observed metallicity distribution function in Fig. 2 peaks around $0.2 \mathrm{dex}$, which is higher than previous reported solar vicinity MDFs (see e.g., Fuhrmann et al. 2017). A possible ignored bias towards more metal-rich objects in the sample selection could be pulling the percentage of possible migrators to higher values. Among the entire distribution, our churned candidates with $[\mathrm{M} / \mathrm{H}]>+0.1$ comprise around $17 \%$ of the sample. If we constrain the number of migrators to only stars with $[\mathrm{M} / \mathrm{H}]>+0.25$, the global percentage decreases to $8 \%$ of the sample.

In particular, we analysed the possible age trends in the radial changes $\left(\Delta R_{g}=R_{g}-R_{\text {birth }}\right)$ associated to churning. For each assumed ISM abundance gradient separately, Fig. 7 shows the $\Delta R_{g}$ as a function of stellar age for the churned candidates (see Fig. 6). The migrated SMR stars cover a wide range of ages from $\sim 4$ to 12 Gyr. As a consequence of the $R_{\text {birth }}$ estimation proxy (zero point fixed at $\operatorname{ISM}_{[\mathrm{M} / \mathrm{H}]}\left(R_{\odot}\right)=0.0 \mathrm{dex}$ ), the flatter the applied ISM gradient, the larger the $\Delta R_{g}$. The respective slopes and errors were derived by the Theil-Sen linear regression estimator. We observe that the general trend is slightly negative with stellar age, becoming steeper as the assumed ISM gradient flattens (see right-hand panel). In addition, we obtain different $\Delta R_{g}$ values based on the zero-point expression from the analysis of Genovali et al. (2014), but their study also reveals a shallow neg- ative gradient with stellar age. As a consequence, the zero-point assumption has a direct effect on the value derived for migration distance, but not on the observed general trend with age. For instance, if we assume a higher zero point (e.g., $\operatorname{ISM}_{[\mathrm{M} / \mathrm{H}]}\left(R_{\odot}\right)=$ $0.1 \mathrm{dex})$, the radial migration estimate decreases by almost half for a star with $[\mathrm{M} / \mathrm{H}]=+0.25$.

Additionally, the application of a fixed zero point for every star may introduce a bias in the $\Delta R_{g}$ estimate, in particular for old stars, because $\operatorname{ISM}_{[\mathrm{M} / \mathrm{H}]}\left(R_{\odot}\right)$ is expected to have been chemically enriched with time to reach the present-day value used here. To explore the dependence on the zero-point assumption, we applied a more appropriate value for each star according to its age. For this purpose, we selected the observed stars in our sample that lie on the solar annulus $\left(7.5 \leq R_{g}<8.5 \mathrm{kpc}\right)$, and calculated the average metallicity $([\mathrm{M} / \mathrm{H}]=0.02,-0.03,-0.05$, and -0.15 dex) at different age bins ([2-6], [6-8], [8-10], and [10-12] Gyr). We then assumed these values as a proxy of the $\operatorname{ISM}_{[\mathrm{M} / \mathrm{H}]}\left(R_{\odot}\right)$ evolution with time. The adopted trend is consistent with the ISM enrichment of the solar neighbourhood found by Galactic chemical evolution models (e.g., Hou et al. 2000; Schönrich \& McMillan 2017; Minchev et al. 2018), although an accurate $\operatorname{ISM}_{[\mathrm{M} / \mathrm{H}]}\left(R_{\odot}, \tau\right)$ estimate is outside the scope of this paper. Figure 8 shows the observed trend by applying the estimated zero point as a function of the SMR stellar age. For each analysed ISM abundance gradient, we obtained significantly higher $\Delta R_{g}$ values for older SMR stars up to inverting to a positive trend with stellar age. We stress that the selected stars in the solar annulus are likely to be originally born far from their present location. Therefore, a more accurate $\operatorname{ISM}_{[\mathrm{M} / \mathrm{H}]}\left(R_{\odot}, \tau\right)$ estimate might present lower values than the ones shown here, and the impact on the $\Delta R_{g}$ value could be greater than that suggested by our analysis.

Moreover, we explored the influence of a time evolution of the ISM gradient combined with an evolving $\operatorname{ISM}_{[\mathrm{M} / \mathrm{H}]}\left(R_{\odot}\right)$ zero point on the $R_{\text {birth }}$ estimate. For this purpose, we decided to apply a simple toy model based on the assumptions of Minchev et al. (2018): a thin disc formed with an initial metallicity gradient of $\sim-0.15 \mathrm{dex} \mathrm{kpc}^{-1}$, flattening with time to a present-day ISM profile of $\sim-0.07 \mathrm{dex} \mathrm{kpc}^{-1}$ (which is equal to our measured gradient for young stars, shown in Table 1). Our toy model simply assigns these limit ISM gradient values to the youngest $(3.3 \mathrm{Gyr})$ and the oldest (11.8 Gyr) SMR stars in our selected sample. A linear interpolation was then performed to estimate the corresponding ISM metallicity gradient as a function of each SMR stellar age. Figure 9 shows the resulting trend of $\Delta R_{g}$ with stellar age, which has now changed sign compared to the one derived 

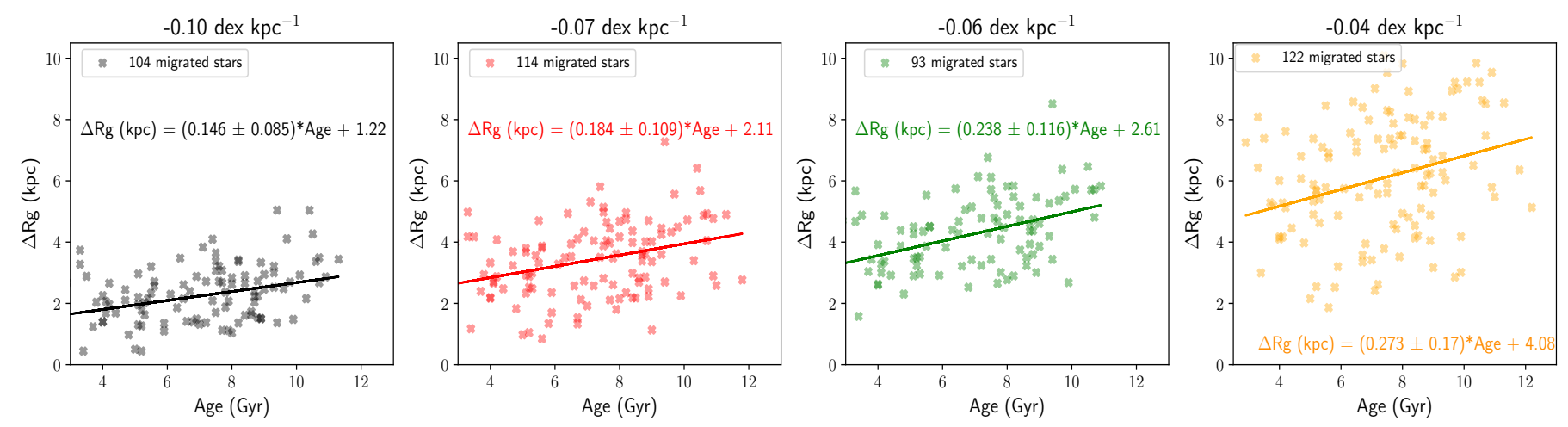

Fig. 8. Same as Fig. 7, but applying a different zero point as a function of the stellar age $\left(\operatorname{ISM}_{[\mathrm{M} / \mathrm{H}]}\left(R_{\odot}, \tau\right)\right)$ in the $R_{\text {birth }}$ estimate (see Sect 3.3).

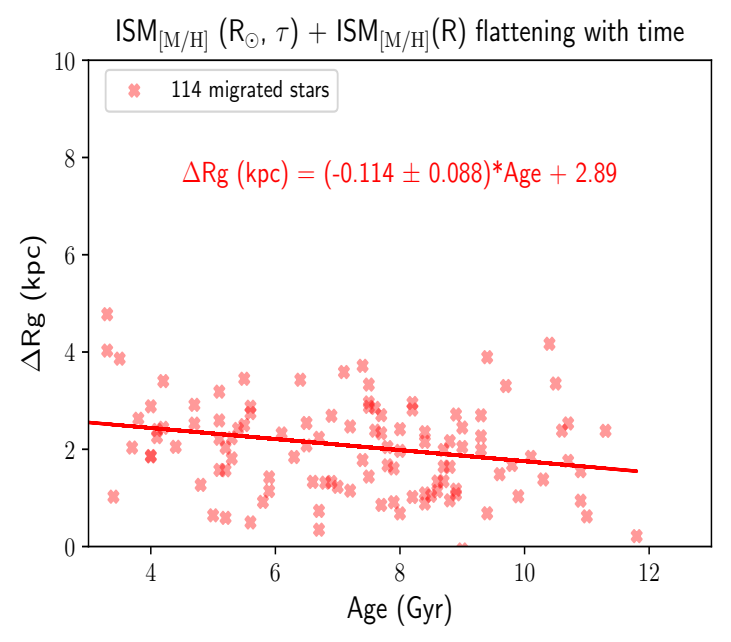

Fig. 9. Estimated covered distance $\Delta R_{g}\left(R_{g}-R_{\text {birth }}\right)$ vs. stellar age for the selected churned SMR stars, applying a different zero point $\left(\operatorname{ISM}_{[\mathrm{M} / \mathrm{H}]}\left(R_{\odot}, \tau\right)\right)$, and a different ISM metallicity gradient as a function of the stellar age (linearly flattening with time from -0.15 to $-0.07 \mathrm{dex} \mathrm{kpc}^{-1}$ between $11.8 \mathrm{Gyr}$ and $3.3 \mathrm{Gyr}$, respectively) in the $R_{\mathrm{birth}}$ estimate.

in Fig. 8, but is similar to the observed trend in our first simple approach shown in Fig. 7.

We would like to highlight the fact that the aim of this analysis is to visualise the strong dependence on the assumptions of the observed radial migration trend with age. An accurate $\mathrm{ISM}_{[\mathrm{M} / \mathrm{H}]}(R)$ determination at any epoch is outside the scope of this paper. For that reason, we assumed a linear one-slope gradient for simplicity to estimate the birth radius of the stars for each analysed case. A more accurate metallicity gradient (e.g., flattens at $R<6 \mathrm{kpc}$ as found by Hayden et al. 2014; Haywood et al. 2019) would probably provide more accurate values (i.e., with no need to go further than $2-3 \mathrm{kpc}$ to find the most metal-rich stars, as shown by the APOGEE results from Hayden et al. 2014, 2015), but the dependence of the general trends on the assumptions of the illustrated model would be similar to those reported here.

In conclusion, our local analysis hints towards a clear, although not necessary predominant, presence of radially migrated stars in the Galactic disc via churning, in agreement with the findings of Kordopatis et al. (2015a). The more realistic scenario illustrated in Fig. 9 (assuming a time evolution of the ISM gradient and the $\operatorname{ISM}_{[\mathrm{M} / \mathrm{H}]}\left(R_{\odot}\right)$ zero point) shows an average $\left\langle\Delta R_{g}\right\rangle=2.03 \pm 0.95 \mathrm{kpc}$ for a broad range of ages
( $3.3 \leq \tau \leq 11.8 \mathrm{Gyr})$. In the same way, we estimate an average $\left\langle\Delta R_{g}\right\rangle=2.06 \pm 0.84 \mathrm{kpc}$ in Fig. 7 (left-most panel) for the case of an ISM gradient similar to the measured one in this work ( $\sim-0.10 \mathrm{dex} \mathrm{kpc}-1$; see Table 1$)$, which is compatible with the measured gradient by Hayden et al. (2014) using APOGEE data (shown in Table 2). This behaviour suggests that an important fraction of stars in the Galactic disc could have also been sensitive to radial changes associated to churning, favouring a scenario where metal-rich stars may come from $2-3 \mathrm{kpc}$ from the Sun. Unfortunately, from an observational point of view, only high-metallicity stars in the solar vicinity can realistically be assumed to be radial migrators. We again mention how complicated it is to accurately measure the radial migration efficiency, and how the interpretation of the reported signatures strongly relies on the model assumptions.

\section{Age-abundance relations}

In this section, we analyse the Galactic disc as a whole, without considering a thin-thick disc dichotomy, to allow a general, parametrised view of the disc evolution with time.

\section{1. $[\mathrm{Mg} / \mathrm{Fe}]$ abundance as a chemical clock}

Figure 10 shows the $[\mathrm{Mg} / \mathrm{Fe}]$ versus age relation colour-coded according to stellar metallicity. The figure clearly shows a significant spread in stellar age at any given $[\mathrm{Mg} / \mathrm{Fe}]$ value, particularly for $[\mathrm{Mg} / \mathrm{Fe}]$ lower than $0.2 \mathrm{dex}$. The covered age range at a fixed $[\mathrm{Mg} / \mathrm{Fe}]$ is generally wider in comparison with the Hayden et al. (2017) analysis of the same sample of stars (see their Fig. 2) using Gaia DR1 data and abundances from Mikolaitis et al. (2017). Furthermore, for stars younger than about $11 \mathrm{Gyr}$, we observe a larger dispersion in the $[\mathrm{Mg} / \mathrm{Fe}]$ abundance $\left(\sigma_{[\mathrm{Mg} / \mathrm{Fe}]} \sim 0.1 \mathrm{dex}\right.$ at a given age $)$ than that suggest by previous studies (e.g., Delgado Mena et al. 2019; Nissen et al. 2017, 2020). This dispersion is correlated with the stellar metallicity and is much more apparent thanks to the unveiled slope of $[\mathrm{Mg} / \mathrm{Fe}]$ with $[\mathrm{M} / \mathrm{H}]$ in Santos-Peral et al. (2020) for metal-rich stars. Indeed, in close agreement with Haywood et al. (2013), the lower envelope is occupied by metalrich stars, while the upper envelope is occupied mainly by more metal-poor stars.

As a follow up, we studied the $[\mathrm{Mg} / \mathrm{Fe}]-$ age trends in different metallicity bins. For $[\mathrm{M} / \mathrm{H}] \geq-0.2$, we find stars with ages from $\sim 3$ to $12 \mathrm{Gyr}$, describing a flat trend in the $[\mathrm{Mg} / \mathrm{Fe}]$-age plane, without a change of the slope in the different metallicity bins. This observed pattern could only be 


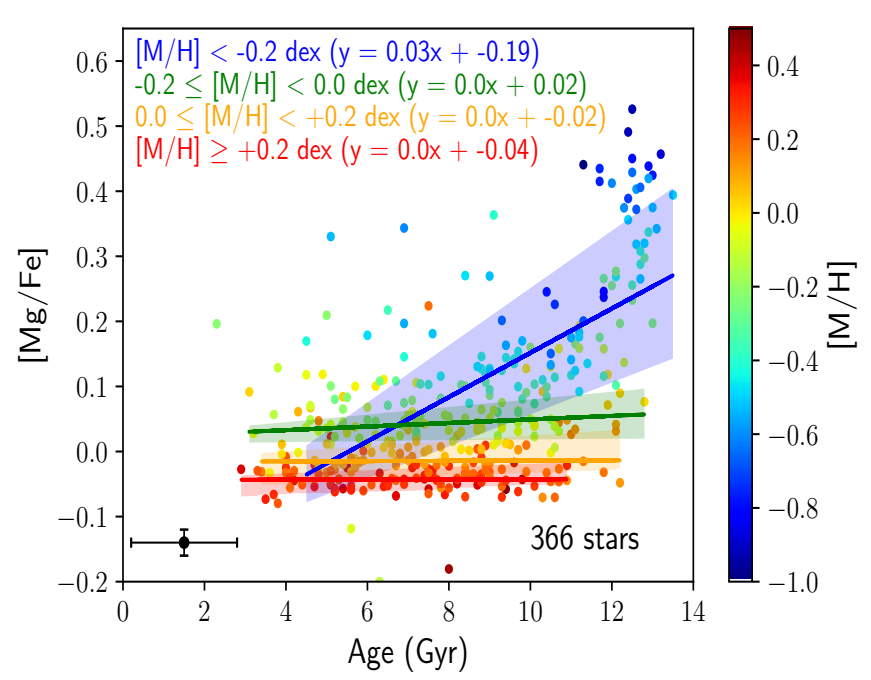

Fig. 10. $[\mathrm{Mg} / \mathrm{Fe}]$ as a function of age for the working sample. The coloured lines correspond to the Theil-Sen linear regression over different metallicity ranges, from metal-poor to metal-rich (from top to bottom). The respective shaded areas span the lower to upper bound of the $95 \%$ confidence interval of the fit. The mean estimated uncertainties for each star value are shown in the bottom-left corner.

explained by chemical evolution models if an important coexistence of different stellar populations in the solar neighbourhood is assumed, with different enrichment histories and birth origins in the Galactic disc (as previously suggested by several studies, e.g., Sellwood \& Binney 2002; Nordström et al. 2004; Fuhrmann 2011; Boeche et al. 2013b; Kordopatis et al. 2015b; Wojno et al. 2016).

For more metal-poor stars $([\mathrm{M} / \mathrm{H}]<-0.2$ dex $)$, we find a linear correlation of $[\mathrm{Mg} / \mathrm{Fe}]$ with age, showing a consistent positive slope value with the results of Delgado Mena et al. (2019) $\left(\sim 0.02\right.$ dex $\mathrm{Gyr}^{-1}$, see their Fig. 7 for a sample of dwarf thin disc stars in the solar neighbourhood), and Ness et al. (2019) ( $\sim 0.03$ dex $\mathrm{Gyr}^{-1}$; see their Fig. 7 for a sample of red clump stars across a wide range of Galactocentric distances). However, these works do not find a clear trend of $[\mathrm{Mg} / \mathrm{Fe}]$ abundances with metallicity in the metal-rich regime such as the one we report for our sample. This slope is also very consistent with the one derived for sulphur by Perdigon et al. (2021).

Finally, we note the presence of young, metal-poor, and high- $[\mathrm{Mg} / \mathrm{Fe}]$ stars, which has also reported in the literature (e.g., Haywood et al. 2013; Martig et al. 2015; Chiappini et al. 2015; Fuhrmann \& Chini 2017a,b; Silva Aguirre et al. 2018; Delgado Mena et al. 2019; Ciucă et al. 2021). Such stars have been suggested to be radially migrated candidates expelled outwards by the Galactic bar (Chiappini et al. 2015), or blue straggler stars produced by mass transfer in binary systems (Jofré et al. 2016; Wyse et al. 2020), which may lead to underestimation of their age.

\subsection{Temporal evolution in the $[\mathrm{Mg} / \mathrm{Fe}]-[\mathrm{M} / \mathrm{H}]$ plane}

Figures 11 and 12 illustrate the $[\mathrm{Mg} / \mathrm{Fe}]$ abundance ratios relative to $[\mathrm{M} / \mathrm{H}]$ for our sample of stars, in different age intervals and Galactic disc locations (top row: inner Galactic disc $R_{g} \leq 7.5 \mathrm{kpc}$; bottom row: outer disc $R_{g}>7.5 \mathrm{kpc}$ ). The bin selection was optimised to allow a significant statistical sample of stars at different radii.
Figure 11 shows that the oldest stellar population $(\tau \geq$ $12 \mathrm{Gyr}$ ) at every radius is located in what has been classically called the thick disc, that is, an $\alpha$-enhanced population (see Sect. 3.1). During this early epoch, we observe rapid chemical enrichment, reaching solar metallicities. In addition, the stars are more centrally concentrated (see Sect. 3.1). Interestingly, 11-12 Gyr ago, a second chemical sequence appears in the outer regions of the Galactic disc, populating the metalpoor low- $[\mathrm{Mg} / \mathrm{Fe}]$ tail and starting at $[\mathrm{M} / \mathrm{H}] \sim-0.8 \mathrm{dex}$ and $[\mathrm{Mg} / \mathrm{Fe}] \sim 0.2 \mathrm{dex}$. This corresponds to the population that has classically been referred to as the thin disc or low- $\alpha$ population, highlighted in the figure by red points. Based on the radial extension of the thick disc sample (see Figs. 4 and 5 in Sect. 3.1), the average $R_{g}$ of this second chemical sequence seems to be significantly larger than the Galactic disc extension at that time $\left(R_{g}<8.5 \mathrm{kpc}\right)$, reaching the outer parts up to $R_{g} \sim 11 \mathrm{kpc}$. Furthermore, those stars are shown to be significantly more metalpoor $([\mathrm{M} / \mathrm{H}] \lesssim-0.4 \mathrm{dex})$ with respect to the coexisting stellar population in the inner parts of the disc (top row). They also show lower $[\mathrm{Mg} / \mathrm{Fe}]$ abundances than the older disc population in the outer parts (lower left panel), although presenting a similar metallicity distribution. This implies a chemical discontinuity in the disc around $11 \mathrm{Gyr}$ ago, suggesting that the new sequence might have followed a different chemical evolution pathway from that followed by the previously formed component, possibly triggered by accretion of metal-poor external gas (e.g., Grisoni et al. 2017; Noguchi 2018; Spitoni et al. 2019; Buck 2020; Palla et al. 2020). However, as a direct comparison with models and simulations is missing in the interpretation of our observational results, we are not able to discard other possible scenarios (e.g., separate evolution of the outer disc).

Figure 12 illustrates the chemical evolution in the last $10 \mathrm{Gyr}$. During this long period of time, the Galactic disc seems to have experienced a slower and more continuous chemical evolution towards more metal-rich and lower $[\mathrm{Mg} / \mathrm{Fe}]$ regimes than the more primitive epochs described in Fig. 11. In addition, the average $z_{\max }$ seems to decrease with age, regardless of the chosen chemical sequence and space location, which could be a signature of a disc settling with time. However, a larger statistical sample would be needed to justify this assumption. Finally, the difference in $z_{\max }$ between both chemical sequences does not allow any relevant conclusion, given the low number of stars and the same followed $z_{\max }$ distribution (the present similarities do not support two distinct disc populations; see Sect. 3.1).

\subsection{Trends with stellar age: relation to radius}

Figure 13 represents the $[\mathrm{Mg} / \mathrm{Fe}]$ versus age (left panel) and the age-metallicity relation (AMR; right panel), in two bins of guiding centre radius: the inner regions $\left(R_{g} \leq 7.5 \mathrm{kpc}\right)$ and the outer regions $\left(R_{g}>7.5 \mathrm{kpc}\right)$. The points correspond to the average abundance estimate for each bin in the (age, $\mathrm{Rg}$ ) space, shown as individual panels in Figs. 11 and 12.

In order to propagate the uncertainties on $[\mathrm{M} / \mathrm{H}],[\mathrm{Mg} / \mathrm{Fe}]$, and age for each star, we performed 1000 Gaussian Monte Carlo realisations within the corresponding error bars of each object. In addition, to include the effect of possible statistical fluctuations in the sampled population, we randomly selected $80 \%$ of the stars for each Monte Carlo realisation, and estimated the average values in age, $[\mathrm{M} / \mathrm{H}]$, and $[\mathrm{Mg} / \mathrm{Fe}]$ for the different bins in age. Finally, the plotted errors correspond to the 16th and 84th percentile values $( \pm 1 \sigma)$ of the resulting distributions of the average age, $[\mathrm{M} / \mathrm{H}]$, and $[\mathrm{Mg} / \mathrm{Fe}]$ abundances. We did not consider 

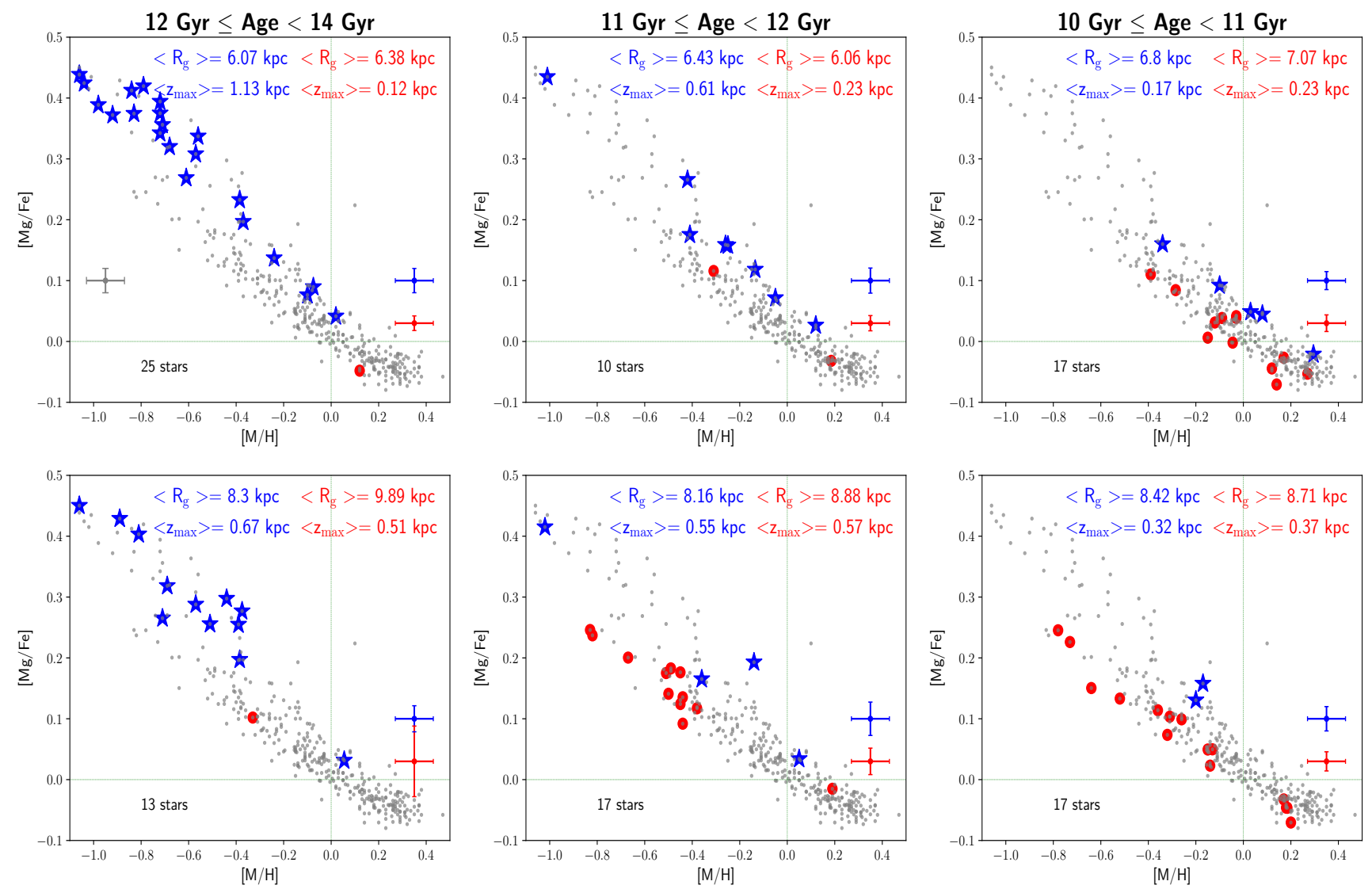

Fig. 11. Distribution of the selected sample in the $([\mathrm{M} / \mathrm{H}],[\mathrm{Mg} / \mathrm{Fe}])$ plane at different ages and locations in the Galaxy $\left(R_{g}\right)$. Two chemical sequences appear for ages younger than $\sim 11-12 \mathrm{Gyr}$, corresponding to the classical thick (blue stars) and thin (red circles) disc components. Each panel corresponds to a bin in the (age, $\left.R_{g}\right)$ space, dividing the Galactic disc into two regions: inner $\left(R_{g} \leq 7.5 \mathrm{kpc}\right.$; top row) and outer $\left(R_{g}>7.5 \mathrm{kpc}\right.$; bottom row). The blue and red crosses in the bottom-right corner of each panel represent the mean estimated errors in $[\mathrm{Mg} / \mathrm{Fe}]$ and $[\mathrm{M} / \mathrm{H}]$ for the thick and thin disc population, respectively, at that particular radius and time. The average $R_{g}$ and $z_{\max }$ for each population are given at the top of each panel. The whole working sample is shown by the dotted grey points.

the uncertainties in the $R_{g}$ estimation because they are negligible (smaller than $0.2 \mathrm{kpc}$, see Sect. 2.4).

Figure 13 shows a rapid chemical enrichment at early epochs (10-14 Gyr), with a sharp increase in metallicity and decrease in $[\mathrm{Mg} / \mathrm{Fe}]$ to solar abundance values. The inner and outer regions describe similar curves, which may indicate that the two samples were drawn from a similar set of stars. We reiterate that the outer disc population does not extend farther than $\sim 8.5 \mathrm{kpc}$ before $~ 11-12$ Gyr.

Subsequently, the appearance of the second Galactic disc sequence separates the average values of the inner and outer disc bins. In particular, the outer bin seems to move away from the initial tracks, with a flatter behaviour in $[\mathrm{M} / \mathrm{H}]$ versus age around $11 \mathrm{Gyr}$. From that epoch, we find two approximately parallel patterns for the inner and outer disc, flattening in the same way with time for the last $10 \mathrm{Gyr}$ of evolution. The measured $[\mathrm{M} / \mathrm{H}]([\mathrm{Mg} / \mathrm{Fe}])$ abundance is shifted towards lower (higher) values for the outer disc at any age, which corresponds to the measured negative (positive) radial gradient in Sect. 3.2. The inside-out scenario for the disc build-up (Matteucci \& Francois 1989; Chiappini et al. 2001), that is, larger timescales for larger radii, which reproduces the observed radial gradients in the disc and the higher surface gas density for star formation in the inner regions, agrees with our observed result. Although our limited sample does not allow us to interpret the disc evolution at different galactocentric distances or smaller bins in $R_{g}$, it is worth noting that the similar observed slopes of the $[\mathrm{Mg} / \mathrm{Fe}]$ and $[\mathrm{M} / \mathrm{H}]$ trends with age may be the signature of a similar chemical evolution at different radii for ages younger than $10 \mathrm{Gyr}$.

Finally, the shift in the abundance space is particularly visible in the outer disc bin and the age interval of 11-12 Gyr (right panel of Fig. 13) because of the appearance of the thin disc sequence, leading to a misalignment between the average abundance value and the error bar for this bin. We find that the Monte Carlo realisations introduced a bias in this particular bin due to the random contamination of younger and older stars in the propagation of the stellar age uncertainties. Compared to the original sample shown in Fig. 11, the inclusion of stars younger than 11 Gyr shifts the metallicity distribution to significantly higher values, while stars older than $12 \mathrm{Gyr}$ do not compensate this bias due to their similar metallicity composition $([\mathrm{M} / \mathrm{H}] \lesssim-0.4 \mathrm{dex})$.

\section{Discussion on the formation and evolution of the Galactic disc}

Different formation and evolution scenarios for the Milky Way disc have been proposed in order to interpret or reproduce the present-day observed chemodynamical trends in the disc. In particular, the chemical bimodality identified in the $([\alpha / \mathrm{Fe}],[\mathrm{M} / \mathrm{H}])$ plane as the thin and thick disc components, and the observed radial abundance gradients, encode information on how the discs have evolved. The main ingredients of disc evolution (mass accretion and radial migration) are examined below in light of our results. 

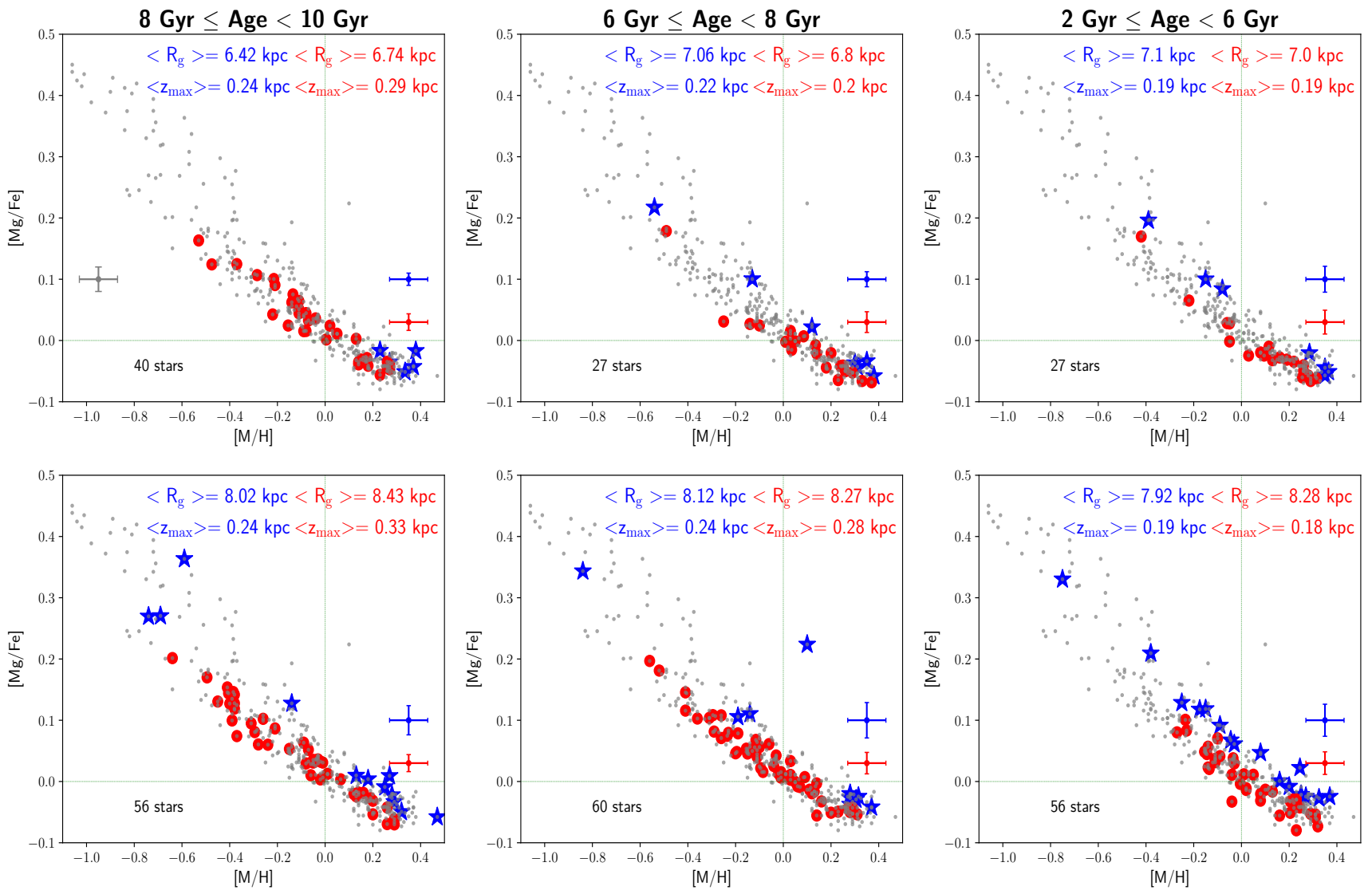

Fig. 12. Same as Fig. 11 but for the younger age intervals.
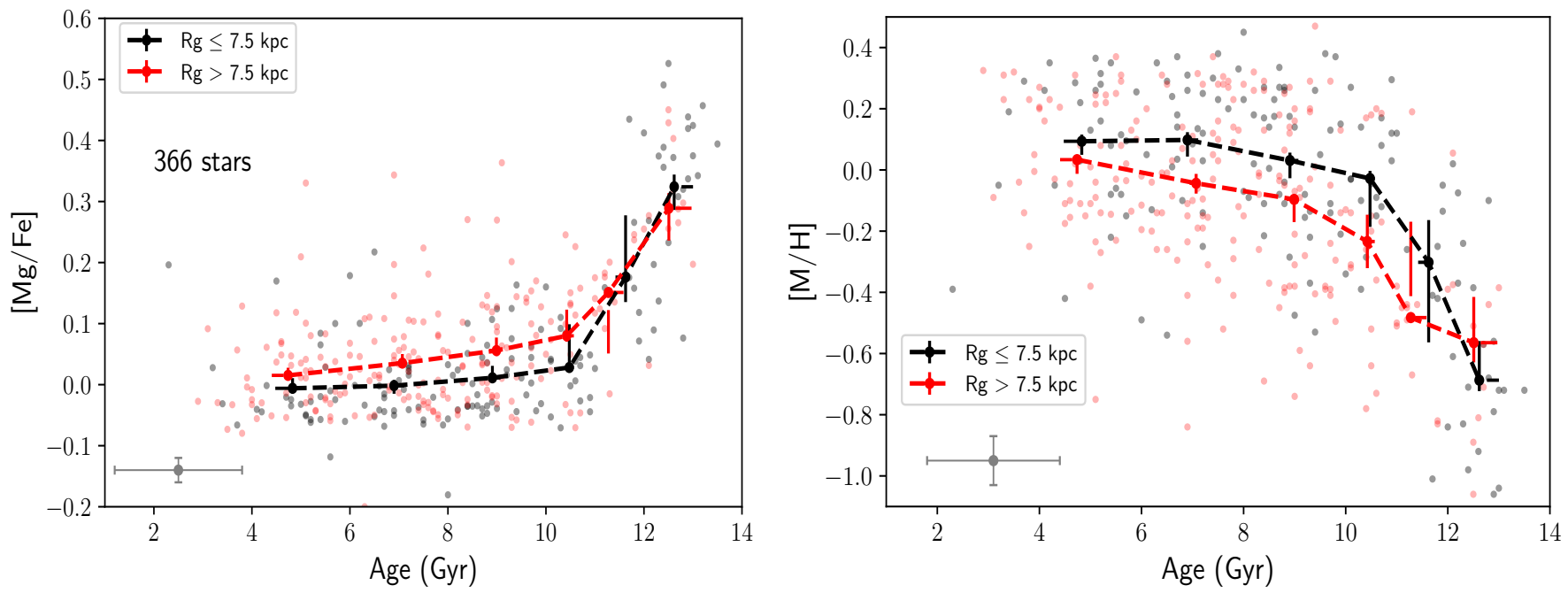

Fig. 13. Stellar distribution in the $[\mathrm{Mg} / \mathrm{Fe}]$ vs. age (left panel) and the $[\mathrm{M} / \mathrm{H}]$ vs. age relation (right panel) for the inner $\left(R_{g} \leq 7.5 \mathrm{kpc}\right)$ and the outer $\left(R_{g}>7.5 \mathrm{kpc}\right)$ regions of the Galactic disc. Their respective trends are indicated by the average abundance value per age bin ([2-6], [6-8], [8-10], [10-11], [11-12] and [12-14] Gyr), together with the error bars in $[\mathrm{Mg} / \mathrm{Fe}],[\mathrm{M} / \mathrm{H}]$, and age. The overall mean estimated errors are shown in the bottom-left corner.

Our results are purely observational and the described interpretation hereafter is based on a revision of literature studies and does not take into account or make direct comparisons with models or simulations. We are aware of this limitation in our interpretation, and a detailed comparison between our data and model results will be performed in a future work, where we hope to draw more robust conclusions.

\subsection{Importance of internal evolutionary processes}

One of the main model assumptions influencing disc evolution is the importance of the Milky Way environment. It is a known fact that the Milky Way is not evolving in isolation, but the role of internal secular processes in shaping the $\alpha$-elements bimodality is difficult to evaluate. To tackle this point, some 
studies have tested the reproducibility of the disc chemodynamical features involving minor quantities of mass accretion and mass loss. In particular, the importance of the following internal processes has been explored: (i) radial flows of gas and radial migration of stars playing a significant role in the stellar distribution (e.g., Schönrich \& Binney 2009; Bovy et al. 2016; Mackereth et al. 2017; Sharma et al. 2021), and (ii) the inflow of metal-poor left-over gas from the outskirts of the disc (Haywood et al. 2019; Khoperskov et al. 2021; Katz et al. 2021) triggered by the formation of the bar and the establishment of the outer Lindblad resonance. For instance, Khoperskov et al. (2021) developed self-consistent chemodynamical simulations of Milky Way-type galaxies that reproduce the observed $\alpha$ bimodality on a long time-scale, after chemical enrichment of the surrounding halo by stellar feedback-driven outflows during the fast thick disc formation.

Our results present a clear $[\mathrm{Mg} / \mathrm{Fe}]$ chemical distinction between both Galactic disc sequences in the metallicity regime $[\mathrm{M} / \mathrm{H}] \leq-0.3 \mathrm{dex}$, and a general decreasing trend even at supersolar metallicities $([\mathrm{M} / \mathrm{H}]>0)$. We find that the oldest observed population $(\tau>12 \mathrm{Gyr}$ ) constitutes the majority of the more metal-poor high-[Mg/Fe] stars, in agreement with the results of previous observational studies of the local disc (e.g., Fuhrmann 2011; Haywood et al. 2013; Hayden et al. 2017), where a temporal transition between the high- and low- $[\mathrm{Mg} / \mathrm{Fe}]$ populations was reported as being due to two different star formation epochs. Furthermore, we identified the presence of the metal-poor low- $[\mathrm{Mg} / \mathrm{Fe}]$ stellar population in the outer regions of the Galactic disc (up to $R_{g} \sim 11 \mathrm{kpc}$ ) around 10-12 Gyr ago (old metal-poor low-alpha disc stars have also been found before; e.g., Haywood et al. 2013; Delgado Mena et al. 2019; Buder et al. 2019). These stars appear significantly more metalpoor $([\mathrm{M} / \mathrm{H}] \lesssim-0.4 \mathrm{dex})$ than the coexisting stellar population in the inner parts of the disc. For the next $10 \mathrm{Gyr}$ of evolution up to the present day, we do not observe significant differences in the chemical enrichment of the inner and outer regions, both presenting similar slopes with respect to the stellar age.

We evaluated our results considering the above-mentioned works that test dominant internal evolution processes. First, regarding radial migration, our estimates in Sect. 3.3 support an important efficiency of the churning process, although the actual amplitude of the induced radial changes remains uncertain. Second, the scenario whereby the metal-poor thin disc stars originate from an inflow of gas from the outskirts of the disc (Haywood et al. 2013, 2019) could be challenged by the similar continuous behaviour of the chemical tracks with radius, which do not show enrichment discontinuities between the internal and the external regions. Indeed, as mentioned above, after the appearance of the low- $[\mathrm{Mg} / \mathrm{Fe}]$ sequence around $10-12 \mathrm{Gyr}$ ago, our results show similar chemical evolution trends $([\mathrm{Mg} / \mathrm{Fe}]$ vs. age, and AMR) for the inner and the outer Galactic disc; they describe separated patterns, where the measured metallicity is always higher in the inner regions at any age, describing a negative radial gradient. Although our local sample does not allow us to accurately study the disc at different Galactocentric distances, as in the recent works by Haywood et al. (2019) and Katz et al. (2021), the observed behaviour may indicate that the stellar Galactic disc has likely followed the same chemical evolution path but at a different radius, without any apparent radial discontinuity. This does not preclude a separate evolution of the external disc before this secondary phase 10-12 Gyr ago, but it would imply a change in the radial dependence of the chemical evolution, which would need to be explained. Further analysis with a larger disc stellar sample, beyond the solar vicinity region, is requied in order to draw more robust conclusions.

\subsection{Mass accretion}

Recent analyses based on Gaia DR2 data have revealed that the Galaxy is perturbed from equilibrium, finding evidence that the Galactic disc can be strongly perturbed by external interactions with satellite galaxies (Antoja et al. 2018; Laporte et al. 2019; Gaia Collaboration 2021, latter work with current EDR3 data), affecting its dynamics and star formation history, although there is no general consensus on the interpretation (see also Khoperskov et al. 2019; Bennett \& Bovy 2021). In line with these findings, Helmi et al. (2018) and Belokurov et al. (2018) (see also Haywood et al. 2018) found clear signatures of a major accretion event by a massive satellite approximately $10 \mathrm{Gyr}$ ago, the so-called Gaia-Enceladus/Sausage, which seems to be considered as a key piece of the puzzle. In this context, models invoking a steady Galactic star formation history seem to neglect a very important ingredient of Milky Way history: mass accretion events.

A variety of disc evolution scenarios allow an infall of mass from the Milky Way environment. This includes chemical evolution models predicting infall of gas on long timescales (Chiappini et al. 1997; Spitoni et al. 2020), and cosmological

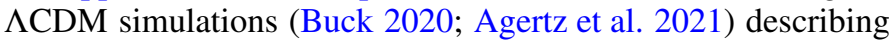
mass accretion through mergers of satellites and gas. In these models, the disc formation goes through two separated star formation epochs driven by two main gas-accretion episodes of extragalactic pristine gas, triggering two different chemical evolution pathways (e.g., Chiappini et al. 1997; Grisoni et al. 2017, 2018; Noguchi 2018; Spitoni et al. 2019; Buck 2020; Palla et al. 2020; Montalbán et al. 2021).

Recent cosmological hydrodynamical simulations developed by Buck (2020) reproduce the bimodal sequences in the $[\alpha / \mathrm{Fe}]$ versus $[\mathrm{Fe} / \mathrm{H}]$ plane as a consequence of a gas-rich merger during the evolution of the Galaxy. In agreement with our results, their models recreate a formation scenario where the high- $\alpha$ sequence evolves first in the early galaxy, pre-enriching the ISM to high metallicities before the appearance of the low- $\alpha$ sequence. The second low- $\alpha$ sequence is formed after the gasrich merger, which provides metal-poor gas, diluting the ISM metallicity. In all simulations, these latter authors observed an old (younger), more radially concentrated (extended) high (low)$\alpha$ disc, compatible with our results. Additionally, the recent work by Agertz et al. (2021), using cosmological zoom-in simulations of a Milky Way-mass disc galaxy, found a similar connection between a last major merger at $z \sim 1.5$ and the formation of an outer, metal-poor, low- $\alpha$ gas disc around the inner, in-situ metalrich galaxy containing the old high- $\alpha$ stars.

There is an apparent overlap in time and metallicity between the reported formation of the outer metal-poor low- $[\mathrm{Mg} / \mathrm{Fe}]$ disc in our results and the signature of a major merger by a massive satellite found in the literature (Helmi et al. 2018; Belokurov et al. 2018; Myeong et al. 2018; Di Matteo et al. 2019; Gallart et al. 2019; Fernández-Alvar et al. 2019; Belokurov et al. 2020; Feuillet et al. 2020; Naidu et al. 2020; Kordopatis et al. 2020). Therefore, our results seem to be consistent with the arrival of a pristine gas-rich merger after the formation of the pre-existing old high- $[\mathrm{Mg} / \mathrm{Fe}]$ population, which may have been diluted at different Galactic radii, leading to the formation of the second $[\mathrm{Mg} / \mathrm{Fe}]$ sequence on longer timescales. In other words, we find that the Galaxy could have already formed a significant population of thick-disc stars before 
the infall of the satellite galaxy Gaia-Enceladus/Sausage, which could have triggered the formation of the thin-disc sequence more than 10 Gyr ago.

Confirmation of this proposed scenario could be provided by a comparison with chemical evolution models. For instance, the recent work by Palla et al. (2020), who applied new updated twoinfall models to reproduce observational data from the APOGEE survey, discuss several cases where the thick and the thin disc are formed by enriched infall episodes including the chemical composition of Gaia-Enceladus/Sausage, finding that this satellite merger could have only contributed to the formation of the thick disc. Their best model suggests a delayed two-infall model (gap of around $\sim 3.25 \mathrm{Gyr}$ between the formation of the two discs), in agreement with a previous study by Spitoni et al. (2019, delay of $\sim 4.3 \mathrm{Gyr}$ ), with a variable star formation efficiency and the presence of radial gas flows during evolution of the Galactic disc. Further, detailed analyses are currently being performed by these latter authors, who are directly comparing their models with our data. The obtained results will be described in a future paper (Palla et al. in prep.).

Our interpretation of our observations is that a pre-existing old high- $[\mathrm{Mg} / \mathrm{Fe}]$ population set the initial chemical conditions in the inner regions $\left(R_{g}<8.5 \mathrm{kpc}\right)$. As a higher surface density towards the Galactic centre is expected already at early epochs, the pristine accreted gas from Gaia-Enceladus/Sausage could have been diluted with a progressively increasing amount of in situ enriched ISM gas towards the disc inner regions. Beyond the limiting radius of the more compact early disc, the infalling gas could have triggered the formation of stars with a chemical composition equal to the accreted one. This scenario would be consistent with the observed chemical similarities between the more metal-rich Gaia-Enceladus/Sausage stars and the metalpoor thin disc population, which are located in neighbouring regions of the $[\mathrm{Mg} / \mathrm{Fe}]$ versus $[\mathrm{M} / \mathrm{H}]$ plane (see e.g., Helmi et al. 2018; Myeong et al. 2019; Feuillet et al. 2020). In addition, this scenario would reproduce the observed chemical radial gradients and the similar observed evolution with age in the inner and outer regions.

\subsection{Radial mixing of stars or gas}

The interpretation of the abundance gradient evolution with time might be misleading if an undetermined fraction of stars were actually born far from their present location (Magrini et al. 2009). Radial stellar migration can decouple the observed evolution of stellar chemical gradients from that of the gradients found in the ISM (Roškar et al. 2008; Pilkington et al. 2012; Schönrich \& McMillan 2017).

To account for this uncertainty, our analysis of Sect. 3.3 considers the impact of different zero points and metallicity gradients in the estimates of stellar migration via churning. All the considered radial distributions imply a significant fraction of churned stars in the solar neighbourhood, even considering a slightly super-solar metallicity for the present-day abundance (e.g., Genovali et al. 2014). Nevertheless, an important uncertainty remains in the amplitude of the induced radial changes. Indeed, some of the explored scenarios in Figs. 7-9 imply $R_{g}$ variations smaller than $1-2 \mathrm{kpc}$ through the entire disc evolution. We can therefore not rule out the possibility that the observed evolution of stellar radial gradients with age is a good first approximation of the actual time-evolution of the ISM. From a dynamical point of view, the possibility of an intense radial migration inducing only small changes in $R_{g}$ of the stellar distribution is out of the scope of the present paper. A similar analysis by Feltzing et al. (2020) also shows an effective radial migration where some fraction of stars have not moved radially since they formed. It is also worth noting that the effect of radial migration on the $[\alpha / \mathrm{Fe}]$ versus $[\mathrm{M} / \mathrm{H}]$ plane deserves further investigation.

Finally, radial gas flows could occur in a scenario invoking gas accretion to explain the low- $\alpha$ sequence formation. It is indeed physically plausible that the infalling gas could have had lower angular momentum than the circular motions in the disc, inducing a radial gas inflow towards the inner parts (Spitoni \& Matteucci 2011; Schönrich \& McMillan 2017). This would contribute to the dilution of the pre-enriched ISM abundance, shaping the radial gradient 10-12 Gyr ago.

\subsection{Chemical enrichment efficiency and star formation rate}

At early times $(10 \leq \tau<14 \mathrm{Gyr})$, the observed high-[Mg/Fe] population in our analysis presents a steep age-metallicity relation, showing an active chemical enrichment, pre-enriching the ISM up to the metal-rich regime before the second low- $[\mathrm{Mg} / \mathrm{Fe}]$ sequence started to form. These observations are consistent with chemical evolution models predicting an early and fast thick disc formation from a massive gaseous disc - mainly supported by turbulence- with a strong star formation rate (SFR) and well-mixed ISM, likely triggered by numerous mergers and accretion events (Haywood et al. 2013; Snaith et al. 2014; Nidever et al. 2014) or due to the rapid collapse of the halo (e.g., Boissier \& Prantzos 1999; Pilkington et al. 2012; Kordopatis et al. 2017).

In the last $10 \mathrm{Gyr}$, our analysis shows a flattening with time of the chemical enrichment (cf. Fig. 13), with a similar slope for the inner and outer regions. This suggests a lower star formation rate with no major radial dependence in the absence of an efficient radial migration. If a radius-dependent SFR were at play, with an increasing SFR with decreasing radius (as expected if the gas density decreases outwards), the radial migration would likely compensate its effects to mimic a similar chemical enrichment with age at all radii. Indeed, as the stellar density is higher in the inner Galaxy, there are more stars migrating outwards than inwards. Therefore, in the presence of active radial migration a radius-dependent SFR is compatible with our observations. In this framework, the evolution of radial gradients with time will also be affected, inducing a progressive flattening.

\section{Summary}

We carried out a detailed chemodynamic analysis of a sample of 494 MSTO stars in the solar neighbourhood $(d<300$ pc with respect to the Sun) observed at high spectral resolution $(R=$ $115000)$ and parametrised within the context of the AMBRE Project. From this sample, we selected 366 stars for which we estimated accurate ages and kinematical and dynamical parameters using the accurate astrometric measurements from the Gaia DR2 catalogue.

For the analysis, we used the $[\mathrm{Mg} / \mathrm{Fe}]$ abundances from Santos-Peral et al. (2020). Thanks to careful treatment of the spectral continuum placement for the different stellar types and each particular $\mathrm{Mg}$ line separately, we obtained a significant improvement of the precision of abundances for the metal-rich population $([\mathrm{M} / \mathrm{H}]>0$ dex $)$, observing a decreasing trend in the $[\mathrm{Mg} / \mathrm{Fe}]$ abundance even at supersolar metallicites. In contrast, preliminary observational studies of the solar neighbourhood revealed a flattened trend. Therefore, in the present work we studied their impact on the reported chemodynamical features (radial chemical abundance gradients, role of radial migration or 
'churning', and age-abundance relations), and therefore on the interpretation of the Galactic disc evolution.

First of all, we explored the present-day chemical abundance distribution $([\mathrm{Mg} / \mathrm{Fe}],[\mathrm{M} / \mathrm{H}])$ in the thin disc at different Galactic radii and ages, after applying a chemical definition to avoid possible thick-disc contamination:

Radial chemical trends. We adopted the guiding centre radius $\left(R_{g}\right)$ as an estimate of the current stellar Galactocentric radius $\left(R_{\mathrm{GC}}\right)$ to derive the radial abundance gradients. We find a consistent negative gradient of $-0.099 \pm$ $0.031 \mathrm{dex} \mathrm{kpc}^{-1}$ for $[\mathrm{M} / \mathrm{H}]$ and a slightly positive gradient of $+0.023 \pm 0.009 \mathrm{dex} \mathrm{kpc}^{-1}$ for the $[\mathrm{Mg} / \mathrm{Fe}]$ abundance. The observed steeper $[\mathrm{Mg} / \mathrm{Fe}]$ gradient than that found in the literature is a major consequence of the Santos-Peral et al. (2020) improvement of the metal-rich spectral analysis. In the framework of the time-delay model, this result for the thin disc could be explained by an inside-out formation with a steeper decrease in the star formation efficiency with radius, enhancing the chemical enrichment in the inner regions relative to outer ones.

Stellar migration. Under the assumptions of a set of ISM gradients, we estimated the birth radius $\left(R_{\text {birth }}\right)$ of the stars in our sample, given their $[\mathrm{M} / \mathrm{H}]$, and selected the super-metalrich stars $([\mathrm{M} / \mathrm{H}] \gtrsim+0.1 \mathrm{dex})$. These stars comprise $17 \%$ of the whole sample $(8 \%$ when we constrain to $[\mathrm{M} / \mathrm{H}]>+0.25)$, and cover a wide range of ages from $\sim 4$ to $12 \mathrm{Gyr}$.

In particular, we analysed the possible age trends in the radial changes $\left(\Delta R_{g}=R_{g}-R_{\text {birth }}\right)$ associated to stellar migration via churning. First, we fixed the zero point to the present-day value $\left(\operatorname{ISM}_{[\mathrm{M} / \mathrm{H}]}\left(R_{\odot}\right)=0.0 \mathrm{dex}\right)$, observing a slightly negative trend of $\Delta R_{g}$ with age, which becomes steeper as the assumed ISM gradient flattens. Secondly, we applied an approach to estimate a more appropriate $\operatorname{ISM}_{[\mathrm{M} / \mathrm{H}]}\left(R_{\odot}\right)$ value, which is expected to be chemically enriched with time (Hou et al. 2000; Schönrich \& McMillan 2017). For each analysed ISM abundance gradient, we obtained significantly higher $\Delta R_{g}$ values for old SMR stars up to inverting to a positive trend with stellar age. Finally, we also considered a time evolution of the ISM gradient by applying a simple linear toy model based on an initial metallicity gradient flattening with time (Minchev et al. 2018). The resulting slightly negative trend with stellar age is similar to the observed trend in our first simple approach.

Our analysis showed that regardless of the chosen ISM model, there is a large range of ages for the selected churned stars. This trend suggests that different stars in the Galactic disc could have radially migrated during their lives. However, the radial migration efficiency is very dependent on the adopted ISM gradient (Feltzing et al. 2020).

Secondly, in order to shed light on the history of the Milky Way disc, we explored the time evolution of the observed chemodynamical relations. We considered the Galactic disc as a whole for the evolution analysis:

[Mg/Fe] abundance as a chemical clock. We find a significant spread of stellar ages at any given $[\mathrm{Mg} / \mathrm{Fe}]$ value, particularly for $[\mathrm{Mg} / \mathrm{Fe}]$ higher than $-0.2 \mathrm{dex}$, while for more metal-poor stars we find a linear correlation of $[\mathrm{Mg} / \mathrm{Fe}]$ with age. Furthermore, for stars younger than about $11 \mathrm{Gyr}$, we observe a large dispersion in the $[\mathrm{Mg} / \mathrm{Fe}]$ abundance $\left(\sigma_{[\mathrm{Mg} / \mathrm{Fe}]} \sim 0.1 \mathrm{dex}\right.$ at a given age), which is directly correlated with the stellar metallicity. For $[\mathrm{M} / \mathrm{H}] \geq-0.2$ dex, we find stars with ages from $\sim 3$ up to $12 \mathrm{Gyr}$ old, describing an almost flat trend in the $[\mathrm{Mg} / \mathrm{Fe}]-$ age relation without significant changes of slope among the different metallicity bins.
Chemical discontinuity in the $[\mathrm{Mg} / \mathrm{Fe}]$ versus $[\mathrm{M} / \mathrm{H}]$ plane. We studied the chemical evolution of the $[\mathrm{Mg} / \mathrm{Fe}]$ abundance relative to $[\mathrm{M} / \mathrm{H}]$ at different age intervals and Galactic disc locations: inner $\left(R_{g} \leq 7.5 \mathrm{kpc}\right)$ and outer $\left(R_{g}>7.5 \mathrm{kpc}\right)$. At early epochs (10-14 Gyr), we observed a rapid chemical enrichment, with a sharp increase in metallicity and decrease in $[\mathrm{Mg} / \mathrm{Fe}]$ to solar abundance values, without apparent differences between the inner and outer regions, pointing towards a chemically wellmixed population (Haywood et al. 2013; Nidever et al. 2014; Kordopatis et al. 2017)

Interestingly, around 10-12 Gyr ago, a second sequence appears in the outer regions of the Galactic disc, populating the metal-poor low-[Mg/Fe] tail. The average $R_{g}$ of this second sequence is significantly larger than the Galactic disc extension at that epoch $\left(R_{g}<8.5 \mathrm{kpc}\right)$, reaching the outer parts up to $R_{g} \sim 11 \mathrm{kpc}$. Furthermore, these stars are more metal-poor with respect to the coexisting stellar population in the inner parts, and show lower $[\mathrm{Mg} / \mathrm{Fe}]$ abundances than the prior disc population in the outer parts, although presenting a similar metallicity distribution. This implies a chemical discontinuity in the disc, also observed in the $[\mathrm{Mg} / \mathrm{Fe}]$ and $[\mathrm{M} / \mathrm{H}]$ trends with age, suggesting that the new sequence might have followed a different chemical evolution pathway from that of the previous formed component (Grisoni et al. 2017; Noguchi 2018; Spitoni et al. 2019; Buck 2020). It is worth noting that the present result does not support a spatial discontinuity of the chemical evolution in Galactic radius.

From that epoch, $10 \mathrm{Gyr}$ ago, the Galactic disc seems to have experienced a slower and more continuous chemical evolution. The similar slopes of the $[\mathrm{Mg} / \mathrm{Fe}]$ and $[\mathrm{M} / \mathrm{H}]$ trends with age point to a similar chemical evolution at all radii.

Our analysis sheds new light on the disc evolution process from the perspective of the present-day solar neighbourhood population. This provides a highly precise view, but is limited by the absence of unbiased constraints at different galactic radii.

In this framework, our results favour the rapid formation of an early disc settled in the inner regions $(R<8.5 \mathrm{kpc})$, followed by the accretion of external metal-poor gas possibly related to a major accretion event such as the Gaia-Enceladus/Sausage one 10-12 Gyr ago (Helmi et al. 2018; Belokurov et al. 2018; Di Matteo et al. 2019; Gallart et al. 2019). This would dilute the pre-enriched ISM abundance with a radial dependence induced by the surface density distribution of the early disc and the possible radial inflows of gas. Beyond the limiting radius of the more compact early disc, the infalling gas could have triggered the formation of stars with a chemical composition similar to the accreted Gaia-Enceladus/Sausage population, as supported by the chemical similarities between the more metal-rich GaiaEnceladus/Sausage stars and the metal-poor thin disc population (Mackereth et al. 2019; Feuillet et al. 2020).

In addition, our analysis supports the fact that radial migration via churning was at play in the last $10 \mathrm{Gyr}$ of disc evolution (Kordopatis et al. 2015b), although the actual amplitude of the induced $R_{g}$ changes remains largely uncertain and could be as low as $1-2 \mathrm{kpc}$

Finally, our data support a very fast chemical enrichment at early epochs, slowing down about $10 \mathrm{Gyr}$ ago. The observed similar slopes in the chemical enrichment for the inner and outer regions suggest a possible equilibrium between the radial SFR dependence and the radial migration efficiency.

Acknowledgements. We would like to thank He Zhao and Pedro Alonso Palicio for useful comments and discussions. We thank the anonymous referee for his/her constructive comments, making a considerable contribution to the improvement of the paper. The authors thank Michael Hayden for providing 
derived data from their 2017 paper. This work is part of the $\mathrm{PhD}$ thesis project within the framework of "International Grants Programme" of the Instituto de Astrofísica de Canarias (IAC). P. S. P. would like to thank the Centre National de Recherche Scientifique (CNRS) for the financial support. P. S. P. also acknowledges partial support from the Université Côte d'Azur (UCA). Part of this work was supported by the "Programme National de Physique Stellaire" (PNPS) of CNRS/INSU co-funded by CEA and CNES. E. F. A acknowledges financial support from the French National Research Agency (ANR) funded project "Pristine" (ANR-18-CE31-0017). A. R. B. and P. dL. acknowledge support from the ANR 14-CE33-014-01. This work has made use of data from the European Space Agency (ESA) mission Gaia (https://www . cosmos.esa.int/gaia), processed by the Gaia Data Processing and Analysis Consortium (DPAC, https://www. cosmos.esa.int/web/gaia/dpac/ consortium). Funding for the DPAC has been provided by national institutions, in particular the institutions participating in the Gaia Multilateral Agreement. Most of the calculations have been performed with the high-performance computing facility SIGAMM, hosted by OCA.

\section{References}

Adibekyan, V. Z., Sousa, S. G., Santos, N. C., et al. 2012, A\&A, 545, A32 Agertz, O., Renaud, F., Feltzing, S., et al. 2021, MNRAS, 503, 5826 Anders, F., Chiappini, C., Santiago, B. X., et al. 2014, A\&A, 564, A115 Antoja, T., Helmi, A., Romero-Gómez, M., et al. 2018, Nature, 561, 360 Bailer-Jones, C. A. L., Rybizki, J., Fouesneau, M., Mantelet, G., \& Andrae, R. 2018, AJ, 156, 58

Baratella, M., D'Orazi, V., Carraro, G., et al. 2020, A\&A, 634, A34

Belokurov, V., Erkal, D., Evans, N. W., Koposov, S. E., \& Deason, A. J. 2018, MNRAS, 478, 611

Belokurov, V., Sanders, J. L., Fattahi, A., et al. 2020, MNRAS, 494, 3880 Bennett, M., \& Bovy, J. 2021, MNRAS, 503, 376

Bensby, T., Feltzing, S., \& Lundström, I. 2003, A\&A, 410, 527

Bensby, T., Feltzing, S., Lundström, I., \& Ilyin, I. 2005, A\&A, 433, 185

Bensby, T., Feltzing, S., \& Oey, M. S. 2014, A\&A, 562, A71

Bergemann, M., Ruchti, G. R., Serenelli, A., et al. 2014, A\&A, 565, A89

Binney, J. 2007, Ap\&SS Proc., 3, 67

Boeche, C., Siebert, A., Piffl, T., et al. 2013a, A\&A, 559, A59

Boeche, C., Chiappini, C., Minchev, I., et al. 2013b, A\&A, 553, A19

Boissier, S., \& Prantzos, N. 1999, MNRAS, 307, 857

Bovy, J. 2015, ApJS, 216, 29

Bovy, J., Rix, H.-W., Schlafly, E. F., et al. 2016, ApJ, 823, 30

Bressan, A., Marigo, P., Girardi, L., et al. 2012, MNRAS, 427, 127

Buck, T. 2020, MNRAS, 491, 5435

Buder, S., Lind, K., Ness, M. K., et al. 2019, A\&A, 624, A19

Carlberg, R. G., Dawson, P. C., Hsu, T., \& Vandenberg, D. A. 1985, ApJ, 294, 674

Carrera, R., Bragaglia, A., Cantat-Gaudin, T., et al. 2019, A\&A, 623, A80

Cheng, J. Y., Rockosi, C. M., Morrison, H. L., et al. 2012, ApJ, 746, 149

Chiappini, C., Matteucci, F., \& Gratton, R. 1997, ApJ, 477, 765

Chiappini, C., Matteucci, F., \& Romano, D. 2001, ApJ, 554, 1044

Chiappini, C., Anders, F., Rodrigues, T. S., et al. 2015, A\&A, 576, L12

Ciucă, I., Kawata, D., Miglio, A., Davies, G. R., \& Grand, R. J. J. 2021, MNRAS, 503,2814

de Laverny, P., Recio-Blanco, A., Worley, C. C., \& Plez, B. 2012, A\&A, 544, A126

de Laverny, P., Recio-Blanco, A., Worley, C. C., et al. 2013, Messenger, 153, 18 Delgado Mena, E., Moya, A., Adibekyan, V., et al. 2019, A\&A, 624, A78 De Pascale, M., Worley, C. C., de Laverny, P., et al. 2014, A\&A, 570, A68

Di Matteo, P., Haywood, M., Combes, F., Semelin, B., \& Snaith, O. N. 2013, A\&A, 553, A102

Di Matteo, P., Haywood, M., Lehnert, M. D., et al. 2019, A\&A, 632, A4

Donor, J., Frinchaboy, P. M., Cunha, K., et al. 2020, AJ, 159, 199

Evans, D. W., Riello, M., De Angeli, F., et al. 2018, A\&A, 616, A4

Feltzing, S., Bowers, J. B., \& Agertz, O. 2020, MNRAS, 493, 1419

Fernández-Alvar, E., Tissera, P. B., Carigi, L., et al. 2019, MNRAS, 485, 1745

Feuillet, D. K., Feltzing, S., Sahlholdt, C. L., \& Casagrande, L. 2020, MNRAS, 497, 109

Freeman, K., \& Bland-Hawthorn, J. 2002, ARA\&A, 40, 487

Friel, E. D. 1995, ARA\&A, 33, 381

Frinchaboy, P. M., Thompson, B., Jackson, K. M., et al. 2013, ApJ, 777, L1

Fuhrmann, K. 1998, A\&A, 338, 161

Fuhrmann, K. 2011, MNRAS, 414, 2893

Fuhrmann, K., \& Chini, R. 2017a, ApJ, 834, 114

Fuhrmann, K., \& Chini, R. 2017b, MNRAS, 471, 1888

Fuhrmann, K., Chini, R., Kaderhandt, L., \& Chen, Z. 2017, MNRAS, 464, 2610

Gaia Collaboration (Brown, A. G. A.,) 2018, A\&A, 616, A1

Gaia Collaboration (Antoja, T., ) 2021, A\&A, 649, A8
Gallart, C., Bernard, E. J., Brook, C. B., et al. 2019, Nat. Astron., 3, 932 Genovali, K., Lemasle, B., Bono, G., et al. 2014, A\&A, 566, A37 Genovali, K., Lemasle, B., da Silva, R., et al. 2015, A\&A, 580, A17 Gibson, B. K., Pilkington, K., Brook, C. B., Stinson, G. S., \& Bailin, J. 2013, A\&A, 554, A47

Gilmore, G., \& Reid, N. 1983, MNRAS, 202, 1025

González Hernández, J. I., \& Bonifacio, P. 2009, A\&A, 497, 497

Graczyk, D., Pietrzyński, G., Gieren, W., et al. 2019, ApJ, 872, 85

Green, G. M., Schlafly, E. F., Finkbeiner, D., et al. 2018, MNRAS, 478, 651

Grenon, M. 1989, Ap\&SS, 156, 29

Grenon, M. 1999, Ap\&SS, 265, 331

Grisoni, V., Spitoni, E., Matteucci, F., et al. 2017, MNRAS, 472, 3637

Grisoni, V., Spitoni, E., \& Matteucci, F. 2018, MNRAS, 481, 2570

Halle, A., Di Matteo, P., Haywood, M., \& Combes, F. 2018, A\&A, 616, A86

Hayden, M. R., Holtzman, J. A., Bovy, J., et al. 2014, AJ, 147, 116

Hayden, M. R., Bovy, J., Holtzman, J. A., et al. 2015, ApJ, 808, 132

Hayden, M. R., Recio-Blanco, A., de Laverny, P., Mikolaitis, S., \& Worley, C. C. 2017, A\&A, 608, L1

Hayden, M. R., Bland-Hawthorn, J., Sharma, S., et al. 2020, MNRAS, 493, 2952

Haywood, M., Di Matteo, P., Lehnert, M. D., Katz, D., \& Gómez, A. 2013, A\&A, 560, A109

Haywood, M., Di Matteo, P., Lehnert, M., et al. 2018, A\&A, 618, A78

Haywood, M., Snaith, O., Lehnert, M. D., Di Matteo, P., \& Khoperskov, S. 2019, A\&A, 625, A105

Helmi, A., Babusiaux, C., Koppelman, H. H., et al. 2018, Nature, 563, 85

Henden, A. A., Levine, S., Terrell, D., et al. 2018, in American Astronomical Society Meeting Abstracts \#232, 223.06

Hou, J. L., Prantzos, N., \& Boissier, S. 2000, A\&A, 362, 921

Jofré, P., Heiter, U., Soubiran, C., et al. 2015, A\&A, 582, A81

Jofré, P., Jorissen, A., Van Eck, S., et al. 2016, A\&A, 595, A60

Katz, D., Gomez, A., Haywood, M., Snaith, O., \& Di Matteo, P. 2021, A\&A submitted [arXiv: 2102.02082]

Khoperskov, S., Di Matteo, P., Gerhard, O., et al. 2019, A\&A, 622, L6

Khoperskov, S., Haywood, M., Snaith, O., et al. 2021, MNRAS, 501, 5176

Kordopatis, G., Wyse, R. F. G., Gilmore, G., et al. 2015a, A\&A, 582, A122

Kordopatis, G., Binney, J., Gilmore, G., et al. 2015b, MNRAS, 447, 3526

Kordopatis, G., Amorisco, N. C., Evans, N. W., Gilmore, G., \& Koposov, S. E. 2016, MNRAS, 457, 1299

Kordopatis, G., Wyse, R. F. G., Chiappini, C., et al. 2017, MNRAS, 467, 469

Kordopatis, G., Recio-Blanco, A., Schultheis, M., \& Hill, V. 2020, A\&A, 643, A69

Lacey, C. G. 1984, MNRAS, 208, 687

Laporte, C. F. P., Minchev, I., Johnston, K. V., \& Gómez, F. A. 2019, MNRAS, 485,3134

Lindegren, L., Hernández, J., Bombrun, A., et al. 2018, A\&A, 616, A2

Lynden-Bell, D., \& Kalnajs, A. J. 1972, MNRAS, 157, 1

Mackereth, J. T., Bovy, J., Schiavon, R. P., et al. 2017, MNRAS, 471, 3057

Mackereth, J. T., Schiavon, R. P., Pfeffer, J., et al. 2019, MNRAS, 482, 3426

Magrini, L., Sestito, P., Randich, S., \& Galli, D. 2009, A\&A, 494, 95

Magrini, L., Randich, S., Kordopatis, G., et al. 2017, A\&A, 603, A2

Mannucci, F., Cresci, G., Maiolino, R., Marconi, A., \& Gnerucci, A. 2010, MNRAS, 408, 2115

Martig, M., Rix, H.-W., Silva Aguirre, V., et al. 2015, MNRAS, 451, 2230

Matteucci, F., \& Francois, P. 1989, MNRAS, 239, 885

McMillan, P. J. 2017, MNRAS, 465, 76

Mikolaitis, S.., Hill, V., Recio-Blanco, A., et al. 2014, A\&A, 572, A33

Mikolaitis, Š., de Laverny, P., Recio-Blanco, A., et al. 2017, A\&A, 600, A22

Mikolaitis, Š., Drazdauskas, A., Minkevičiūte, R., et al. 2019, A\&A, 628, A49

Minchev, I., Chiappini, C., \& Martig, M. 2013, A\&A, 558, A9

Minchev, I., Chiappini, C., \& Martig, M. 2014, A\&A, 572, A92

Minchev, I., Anders, F., Recio-Blanco, A., et al. 2018, MNRAS, 481, 1645

Miyamoto, M., \& Nagai, R. 1975, PASJ, 27, 533

Montalbán, J., Mackereth, J. T., Miglio, A., et al. 2021, Nat. Astron., 5, 640

Myeong, G. C., Evans, N. W., Belokurov, V., Sanders, J. L., \& Koposov, S. E. 2018, ApJ, 863, L28

Myeong, G. C., Vasiliev, E., Iorio, G., Evans, N. W., \& Belokurov, V. 2019, MNRAS, 488, 1235

Naidu, R. P., Conroy, C., Bonaca, A., et al. 2020, ApJ, 901, 48

Navarro, J. F., Frenk, C. S., \& White, S. D. M. 1997, ApJ, 490, 493

Ness, M. K., Johnston, K. V., Blancato, K., et al. 2019, ApJ, 883, 177

Nidever, D. L., Bovy, J., Bird, J. C., et al. 2014, ApJ, 796, 38

Nissen, P. E., Silva Aguirre, V., Christensen-Dalsgaard, J., et al. 2017, A\&A, 608, A112

Nissen, P. E., Christensen-Dalsgaard, J., Mosumgaard, J. R., et al. 2020, A\&A, 640, A81

Noguchi, M. 2018, Nature, 559, 585

Nordström, B., Mayor, M., Andersen, J., et al. 2004, A\&A, 418, 989 
Oort, J. H. 1970, A\&A, 7, 381

Palla, M., Matteucci, F., Spitoni, E., Vincenzo, F., \& Grisoni, V. 2020, MNRAS 498, 1710

Perdigon, J., de Laverny, P., Recio-Blanco, A., et al. 2021, A\&A, 647, A162

Pilkington, K., Few, C. G., Gibson, B. K., et al. 2012, A\&A, 540, A56

Recio-Blanco, A., Bijaoui, A., \& de Laverny, P. 2006, MNRAS, 370, 141

Recio-Blanco, A., de Laverny, P., Kordopatis, G., et al. 2014, A\&A, 567, A5

Reddy, B. E., Lambert, D. L., \& Allende Prieto, C. 2006, MNRAS, 367, 1329

Romano, D., Karakas, A. I., Tosi, M., \& Matteucci, F. 2010, A\&A, 522, A32

Roškar, R., Debattista, V. P., Quinn, T. R., Stinson, G. S., \& Wadsley, J. 2008, ApJ, 684, L79

Santos-Peral, P., Recio-Blanco, A., de Laverny, P., Fernández-Alvar, E., \& Ordenovic, C. 2020, A\&A, 639, A140

Schlegel, D. J., Finkbeiner, D. P., \& Davis, M. 1998, ApJ, 500, 525

Schönrich, R., \& Binney, J. 2009, MNRAS, 396, 203

Schönrich, R., \& McMillan, P. J. 2017, MNRAS, 467, 1154

Schönrich, R., McMillan, P., \& Eyer, L. 2019, MNRAS, 487, 3568

Sellwood, J. A., \& Binney, J. J. 2002, MNRAS, 336, 785

Sharma, S., Bland-Hawthorn, J., Binney, J., et al. 2014, ApJ, 793, 51
Sharma, S., Hayden, M. R., \& Bland-Hawthorn, J. 2021, MNRAS, in press, [arXiv:2005.03646]

Silva Aguirre, V., Bojsen-Hansen, M., Slumstrup, D., et al. 2018, MNRAS, 475, 5487

Skrutskie, M. F., Cutri, R. M., Stiening, R., et al. 2006, AJ, 131, 1163

Snaith, O. N., Haywood, M., Di Matteo, P., et al. 2014, ApJ, 781, L31

Spina, L., Ting, Y. S., De Silva, G. M., et al. 2021, MNRAS, 503, 3279

Spitoni, E., \& Matteucci, F. 2011, A\&A, 531, A72

Spitoni, E., Romano, D., Matteucci, F., \& Ciotti, L. 2015, ApJ, 802, 129

Spitoni, E., Silva Aguirre, V., Matteucci, F., Calura, F., \& Grisoni, V. 2019, A\&A, 623, A60

Spitoni, E., Verma, K., Silva Aguirre, V., \& Calura, F. 2020, A\&A, 635, A58

Vincenzo, F., \& Kobayashi, C. 2020, MNRAS, 496, 80

Wojno, J., Kordopatis, G., Steinmetz, M., et al. 2016, MNRAS, 461, 4246

Worley, C. C., de Laverny, P., Recio-Blanco, A., et al. 2012, A\&A, 542, A48

Wyse, R. F. G., Moe, M., \& Kratter, K. M. 2020, MNRAS, 493, 6109

Yoshii, Y. 1982, PASJ, 34, 365

Zwitter, T., Matijevič, G., Breddels, M. A., et al. 2010, A\&A, 522, A54 